\title{
Pure Crystalline Covalent Organic Framework
}

\section{Aerogels}

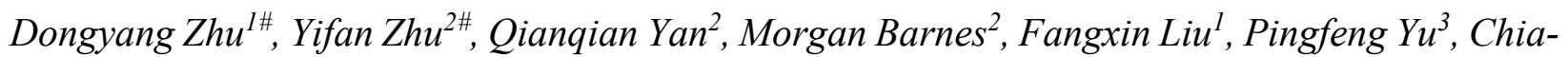
Ping Tseng', Nicholas Tjahjono ${ }^{2}$, Po-Chun Huang ${ }^{1}$, Muhammad M. Rahman ${ }^{2}$, Eilaf Egap ${ }^{2}$, Pulickel M. Ajayan ${ }^{2}$, and Rafael Verduzco ${ }^{1,2 *}$

${ }^{1}$ Department of Chemical and Biomolecular Engineering, Rice University, Houston, Texas 77005, USA

${ }^{2}$ Department of Materials Science and NanoEngineering, Rice University, Houston, Texas 77005, USA

${ }^{3}$ Departmentof Civil and Environmental Engineering, Rice University, Houston, Texas 77005, USA

${ }^{\#}$ D. Zhu and Y. Zhu contributed equally

Corresponding author: Rafael Verduzco

E-mail: rafaelv@ rice.edu 


\section{Synthesis of COF aerogels and powders}

\subsection{Fabrication of TAPB-OMePDA COF aerogels and TAPB-TFPA COF aerogels}

Type I: gelation $+\mathrm{ScCO}_{2}$ dry

TAPB-OMePDA and TAPB-TFPA

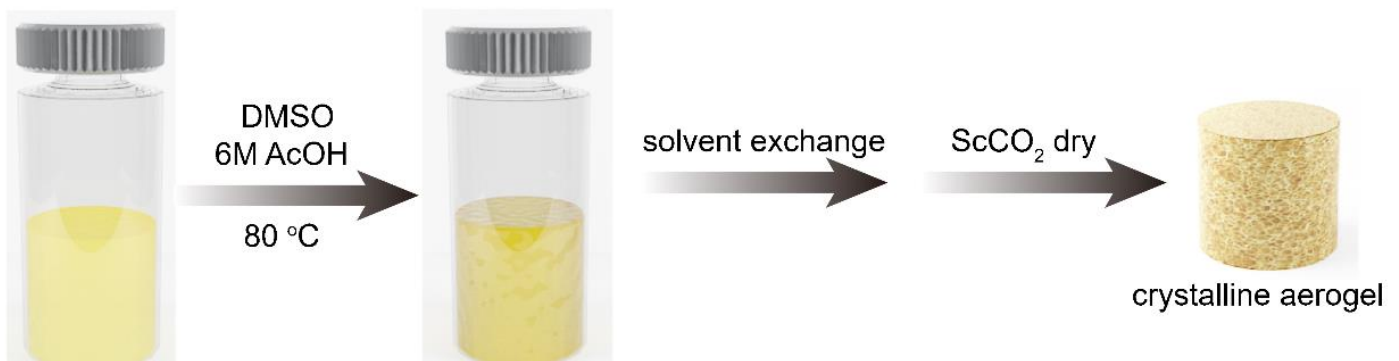

Figure S1. Schematics for synthesis of TAPB-OMePDA COF aerogels and TAPB-TFPA COF aerogels

TAPB-OMePDA COF aerogels and TAPB-TFPA COF aerogels can gain crystallinity directly after reaction in DMSO, solvent exchange and $\mathrm{ScCO}_{2}$ drying. So, the fabrication procedure (see Figure $\mathrm{S} 1$ ) takes three steps: the first step is gelation of COF monomers in DMSO solvent; the second step is solvent exchange to replace all the DMSO solvents in aerogels by using THF, acetone and ethanol; the third step is to dry the wet gels and produce dry aerogels.

In the gelation step, 1,3,5-tris(4-aminophenyl)benzene (TAPB, $21.09 \mathrm{mg}, 0.06 \mathrm{mmol}$ ) and 2,5dimethoxyterephthalaldehyde (OMePDA, $17.48 \mathrm{mg}, 0.09 \mathrm{mmol}$ ) or tris(4-formylphenyl)amine (TFPA, $19.76 \mathrm{mg}, 0.06 \mathrm{mmol}$ ) were dissolved in $1 \mathrm{~mL}$ DMSO in a $4 \mathrm{~mL}$ glass vial. Upon addition of $0.1 \mathrm{ml} 6 \mathrm{M}$ acetic acid, the solution became cloudy immediately and underwent a gelation process within a few minutes. The glass vial was further sealed using Teflon tape to prevent the solvent leakage during the heating process. All samples were heated at $80{ }^{\circ} \mathrm{C}$ for 12 hours.

After reacting for 12 hours, all samples were taken out and cooled down to room temperature. Samples were removed out of the glass vials and immersed in excess THF for three times, excess acetone for three times and finally immerse in excess ethanol for three times. Digital photographs of the wet gels before 
washing were shown in Figure S2. THF has a good solubility and can remove the impurities like unreacted monomers and oligomers. Further solvent exchange using ethanol can replace all the solvents in the previous steps.

After solvent exchange using ethanol, COF aerogels were carefully placed in a tea bag and dried using $\mathrm{ScCO}_{2}$ dryer. Detailed procedures of $\mathrm{ScCO}_{2}$ drying were given in Materials and Methods section, Supporting Information. The weights of the TAPB-OMePDA and TAPB-TFPA COF aerogels are $32.4 \mathrm{mg}$ and $36.3 \mathrm{mg}$. The densities of TAPB-OMePDA and TAPB-TFPA COF aerogels are calculated as $0.032 \mathrm{~g}$ $\mathrm{cm}^{-1}$ and $0.036 \mathrm{~g} \mathrm{~cm}^{-1}$.

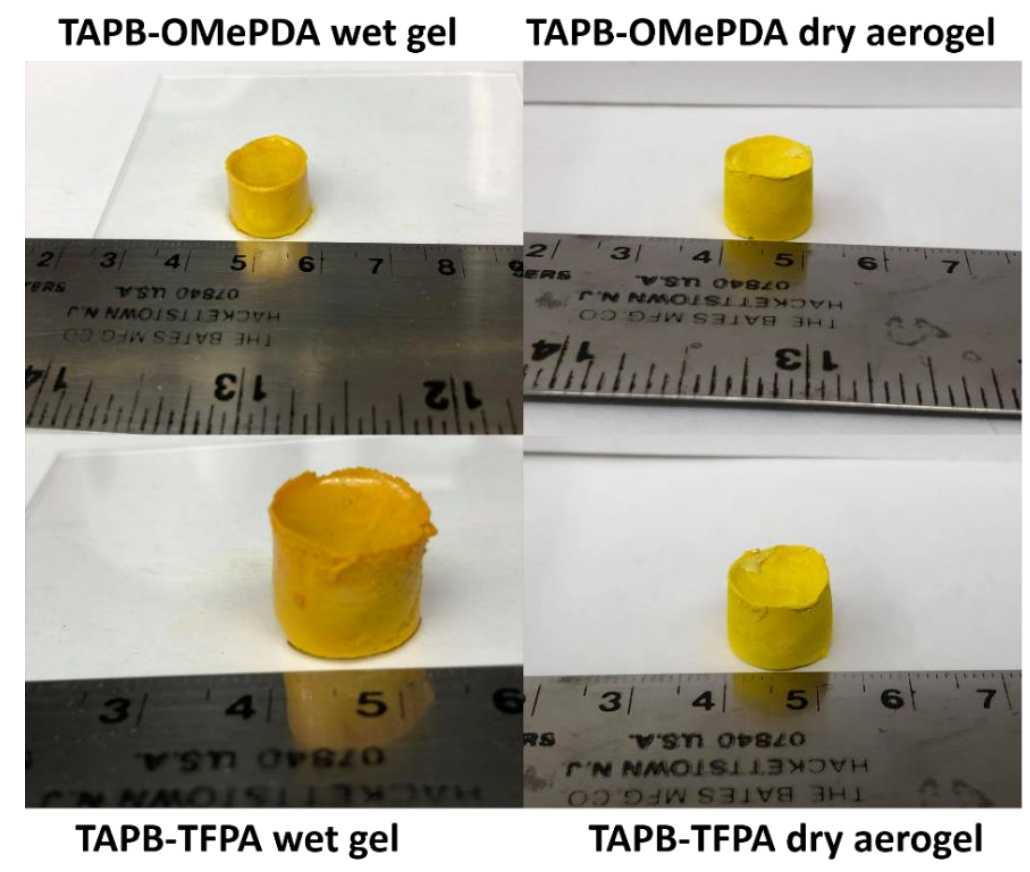

Figure S2. Digital photographs of TAPB-OMePDA and TAPB-TFPA COF wet gels and dry aerogels 


\subsection{Fabrication of TAPA-TFPA, TAPB-PDA, TAPB-BrPDA and BPDA-BTCA COF aerogels}

\section{Type II:gelation+ScCO ${ }_{2}$ dry+reactivation $+\mathrm{ScCO}_{2}$ dry}

TAPA-TFPA, TAPB-PDA, TAPB-BrPDA and BPDA-BTCA



Figure S3. Schematics for synthesis of TAPA-TFPA, TAPB-PDA, TAPB-BrPDA and BPDA-BTCA COF aerogels

TAPA-TFPA, TAPB-PDA, TAPB-BrPDA and BPDA-BTCA COF aerogels cannot gain crystallinity directly after reaction in DMSO, solvent exchange and $\mathrm{ScCO}_{2}$ drying. So, a reactivation procedure is proposed to recover the crystallinity through the dynamic imine exchange reaction. After reactivation, the crystalline COF aerogels are further solvent exchanged and dried using supercritical $\mathrm{CO}_{2}$. The detailed synthesis procedures are shown in Figure S3.

The gelation, solvent exchange and $\mathrm{ScCO}_{2}$ drying process are identical to the procedures for fabricating TAPB-OMePDA and TAPB-TFPA COF aerogels. The monomer amounts for the gelation process are given 
below. TAPA-TFPA COF (TAPA, $0.06 \mathrm{mmol}, 17.42 \mathrm{mg}$; TFPA, $0.06 \mathrm{mmol}, 19.76 \mathrm{mg}$ ); TAPB-PDA (TAPB, 0.06 mmol, 21.09mg; PDA, 0.09 mmol, $12.07 \mathrm{mg}$ ), TAPB-BrPDA (TAPB, 0.06 mmol; BrPDA, $0.09 \mathrm{mmol}, 26.27 \mathrm{mg}$ ) and BPDA-BTCA COF (BPDA, $0.0135 \mathrm{mmol}, 24.87 \mathrm{mg}$; BTCA, $0.09 \mathrm{mmol}, 14.60$ $\mathrm{mg}$ ). Other reaction conditions, solvent exchange and $\mathrm{ScCO}_{2}$ drying process are identical as the synthesis procedures for TAPB-OMePDA COF aerogels.

Amorphous aerogels were reactivated in appropriate solvent systems to gain crystallinity. The reaction conditions in reactivation process are listed in Table $\mathrm{S} 1$. The second-step solvent exchange and $\mathrm{ScCO}_{2}$ drying procedures are similar to the first steps and will not be discussed here. The as-fabricated COF aerogels were shown in Figure S4. The weights of these COF aerogels are $31.2 \mathrm{mg}$ (TAPA-TFPA), 25.7 mg (TAPB-PDA), $41.3 \mathrm{mg}$ (TAPB-BrPDA) and $30.5 \mathrm{mg}$ (BPDA-BTCA), respectively. The densities of these COF aerogels are calculated as $0.031 \mathrm{~g} \mathrm{~cm}^{-1}$ (TAPA-TFPA), $0.026 \mathrm{~g} \mathrm{~cm}^{-1}$ (TAPB-PDA), $0.041 \mathrm{~g} \mathrm{~cm}^{-}$ ${ }^{1}$ (TAPB-BrPDA) and $0.031 \mathrm{~g} \mathrm{~cm}^{-1}$ (BPDA-BTCA), respectively.



Figure S4. Digital photographs of the as-fabricated COF aerogels. The diameters of the COF aerogels are approximately $15 \mathrm{~mm}$. 
Table S1. Reactivation conditions to recover the crystallinity of COF aerogels

\begin{tabular}{|c|l|c|}
\hline COFs & \multicolumn{1}{|c|}{ Reaction conditions } & References \\
\hline TAPA-TFPA & Dioxane/mesitylene/6M AcOH $(5 / 5 / 1, \mathrm{v} / \mathrm{v} / \mathrm{v}), 80^{\circ} \mathrm{C}, 1$ day & 1 \\
\hline TAPB-PDA & Dioxane/mesitylene/6M AcOH $(8 / 2 / 1, \mathrm{v} / \mathrm{v} / \mathrm{v}), 80^{\circ} \mathrm{C}, 1$ day & 2 \\
\hline TAPB-BrPDA & Dioxane/mesitylene/6M AcOH $(8 / 2 / 1, \mathrm{v} / \mathrm{v} / \mathrm{v}), 80^{\circ} \mathrm{C}, 1$ day & none \\
\hline BPDA-BTCA & Dioxane/mesitylene/6M AcOH $(5 / 5 / 1, \mathrm{v} / \mathrm{v} / \mathrm{v}), 80^{\circ} \mathrm{C}, 1$ day & 3 \\
\hline \multicolumn{3}{|c|}{ The reaction conditions were slightly modified. } \\
\hline
\end{tabular}

\subsection{Synthesis of COF powders}

All COF powders were synthesized following the previous reports listed in Table S2.

Table S2. References for the synthesis of COF powders

\begin{tabular}{|l|c|}
\hline \multicolumn{1}{|c|}{ COFs } & References \\
\hline TAPA-TFPA & 1 \\
\hline TAPB-PDA & 2 \\
\hline TAPB-OMePDA & 2 \\
\hline TAPB-BrPDA & No references; reacted in dioxane/mesitylene $(\mathrm{v} / \mathrm{v}, 4 / 1), 120^{\circ} \mathrm{C}, 3$ days \\
\hline TAPB-TFPA & 1 \\
\hline BPDA-BTCA & 3 \\
\hline
\end{tabular}




\section{PXRD analysis}
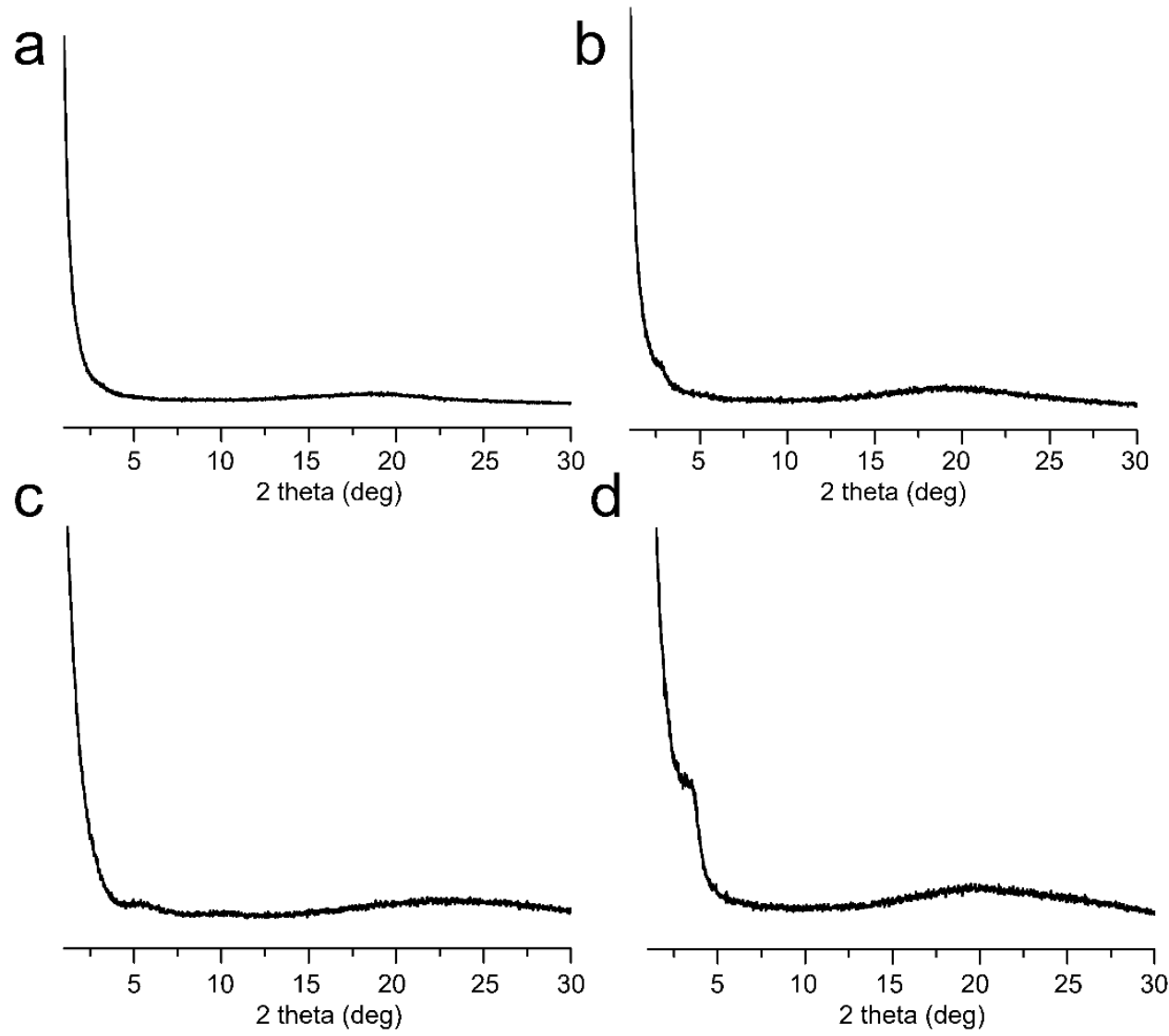

Figure S5. PXRD spectra for four different amorphous COF aerogels before reactivation. (a) TAPATFPA aerogel (b) TAPB-PDA aerogel (c) TAPB-BrPDA and (d) BPDA-BTCA aerogel. 

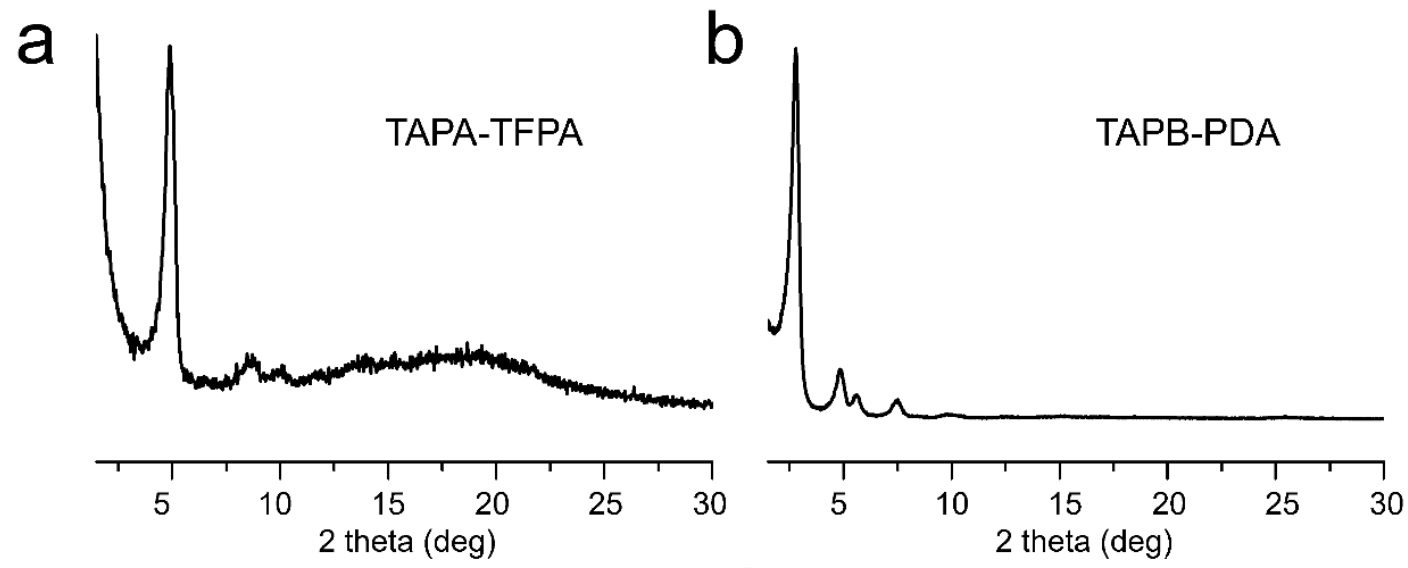

C

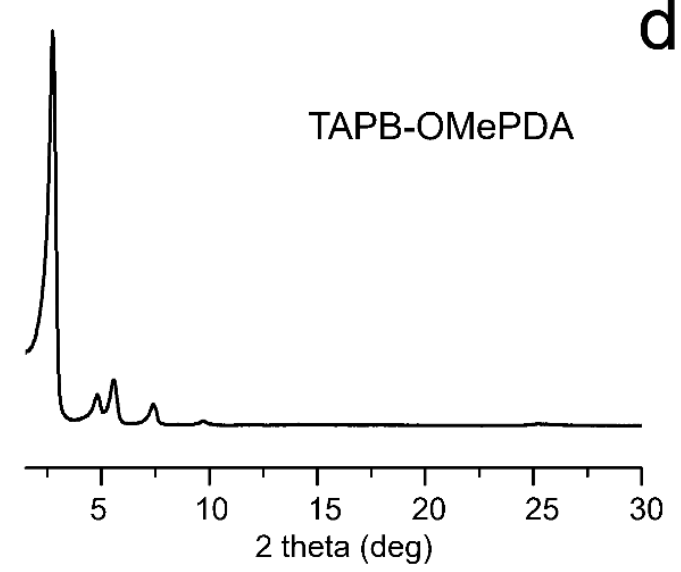

d

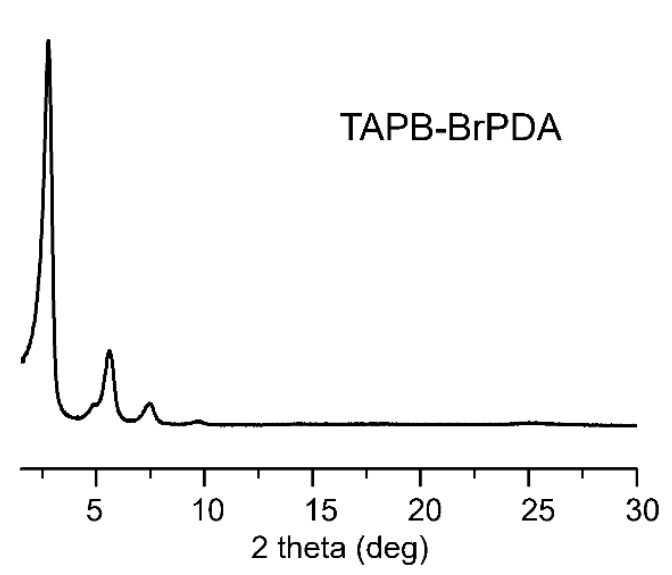

e
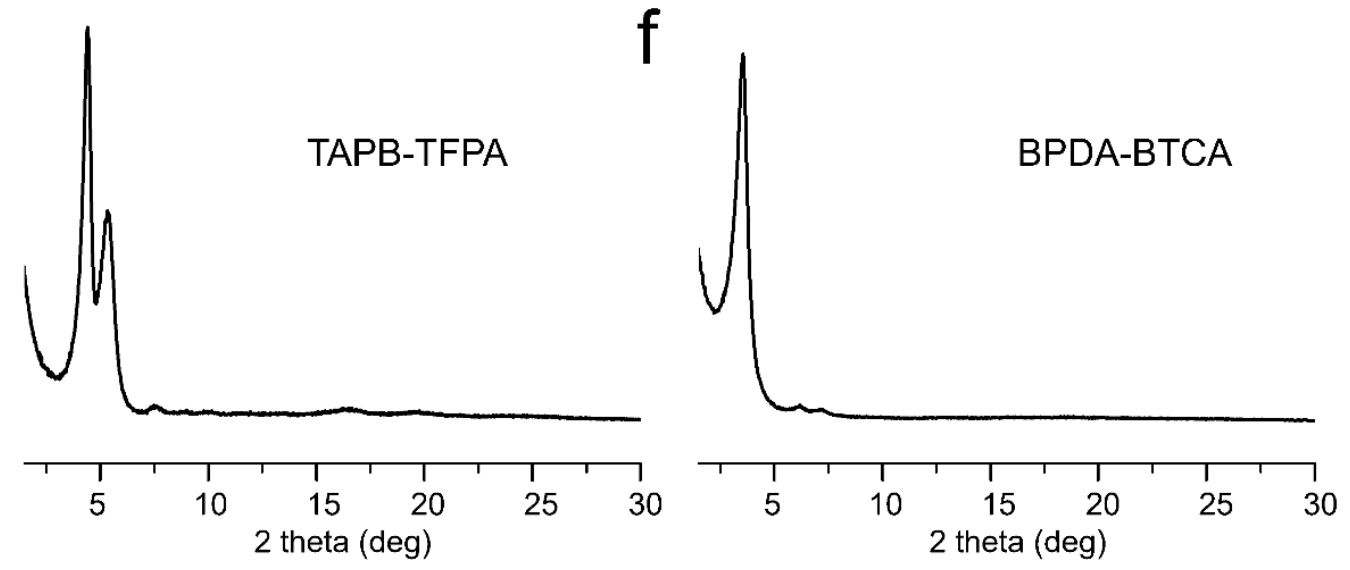

Figure S6. PXRD spectra for six different COF powders. (a) TAPA-TFPA (b) TAPB-PDA (c) TAPBOMePDA (d) TAPB-BrPDA (e) TAPB-TFPA (f) BPDA-BTCA 


\section{FTIR analysis}

a

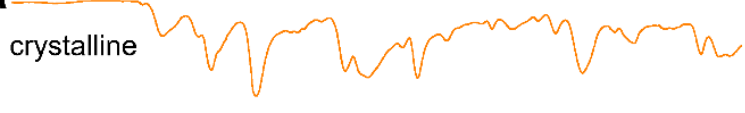

amorphous $\sqrt{ } \sqrt{n} \sqrt{m}$
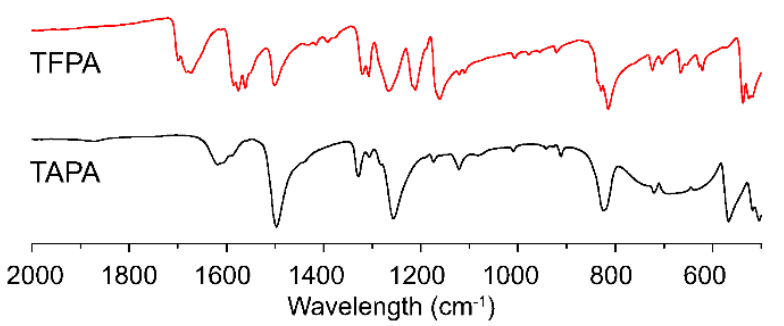

C


e

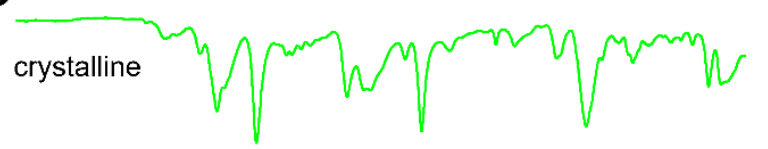

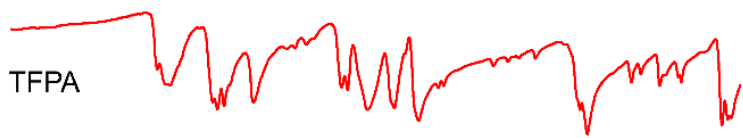
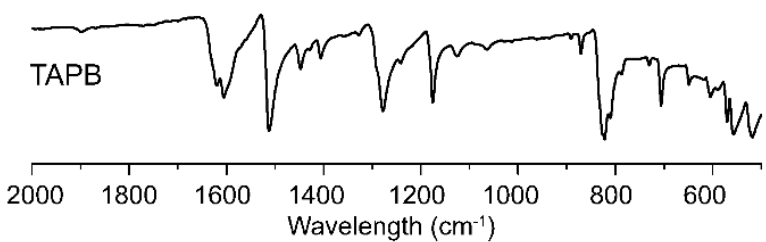

b crystalline W Wrmm

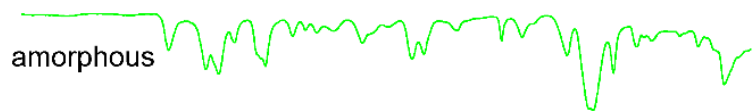
PDA AMW M 2000 1800 1600 $\begin{gathered}1400 \quad 1200 \quad 1000 \\ \text { Wavelength }\left(\mathrm{cm}^{-1}\right)\end{gathered}$ d crystalline $\mathrm{Pr}$ amorphous whrmenrm BrPDA rraphrom TAPB W $\begin{array}{rrrrrrrr}2000 & 1800 & 1600 & 1400 & 1200 & 1000 & 800 & 600\end{array}$ f crystalline Whrmentry amorphous wherving втсA $\sqrt{7 \sqrt{m}} \sqrt{ }$

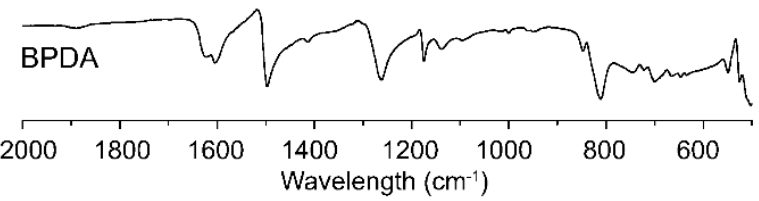

Figure S7. FTIR spectra for various COF monomers, amorphous aerogels and crystalline COF aerogels. (a) TAPA-TFPA (b) TAPB-PDA (c) TAPB-OMePDA (d) TAPB-BrPDA (e) TAPB-TFPA (f) BPDABTCA 


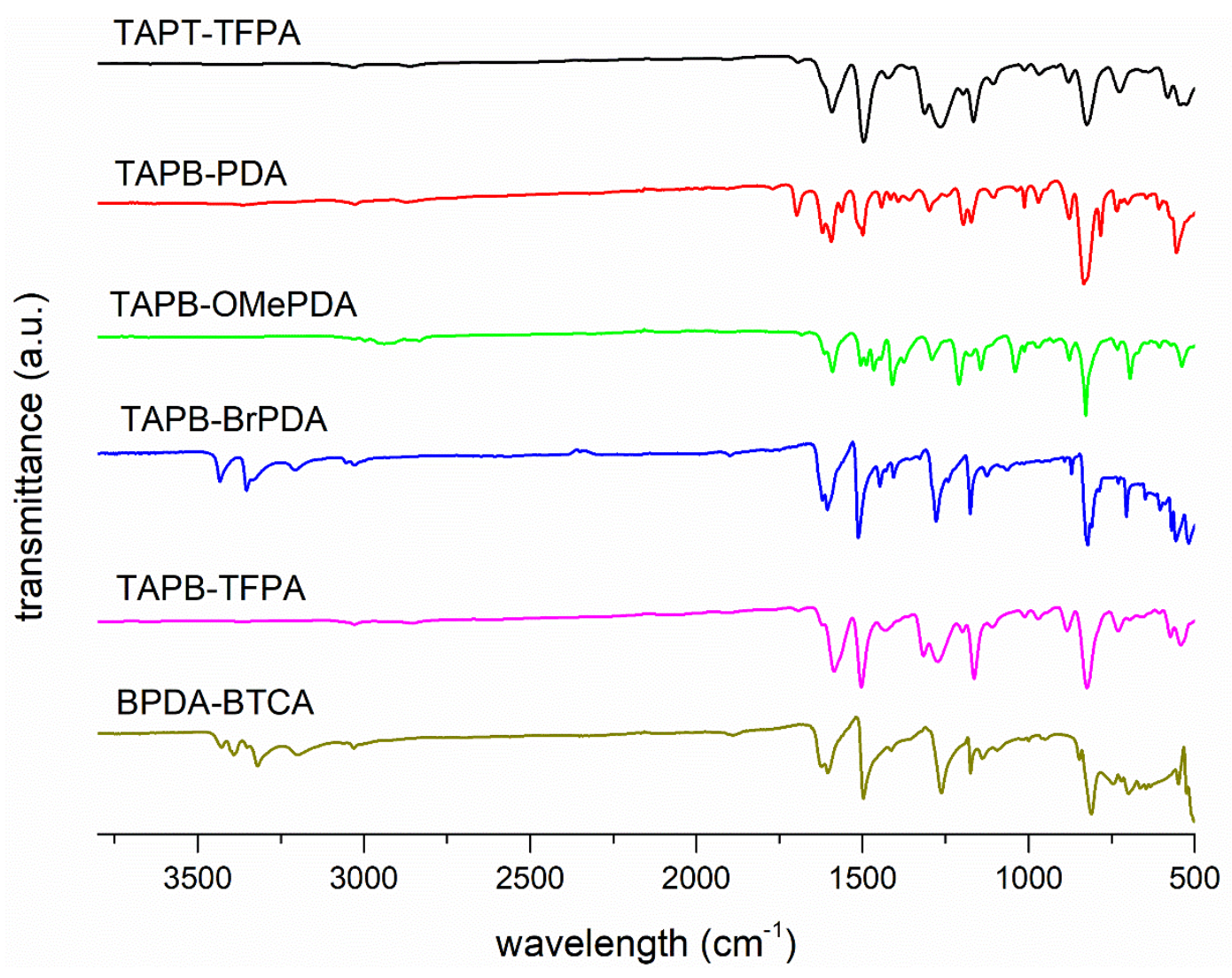

Figure S8. FTIR spectra for six different COF powders. (a) TAPA-TFPA (b) TAPB-PDA (c) TAPBOMePDA (d) TAPB-BrPDA (e) TAPB-TFPA (f) BPDA-BTCA 


\section{Nitrogen sorption analysis}

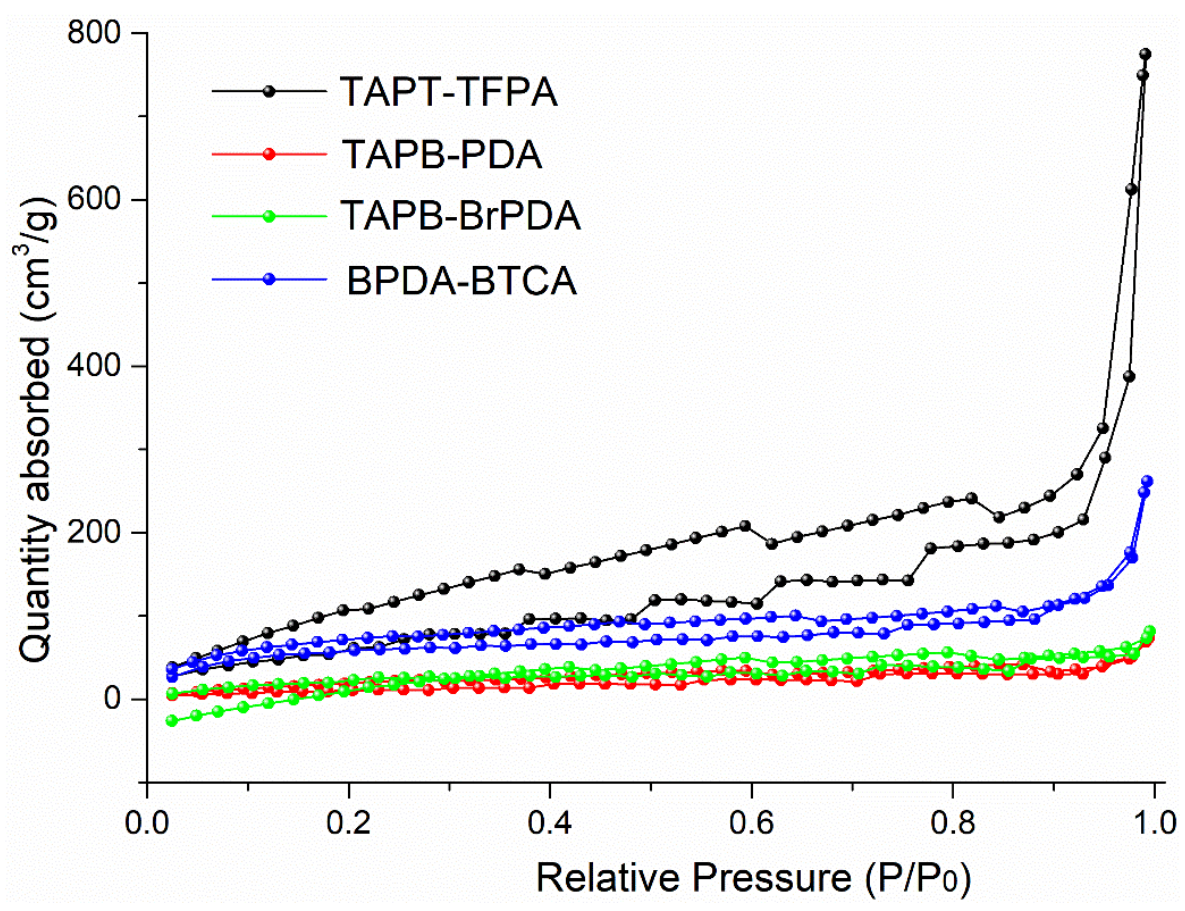

Figure S9. Nitrogen sorption isotherms for four amorphous aerogels before reactivation.
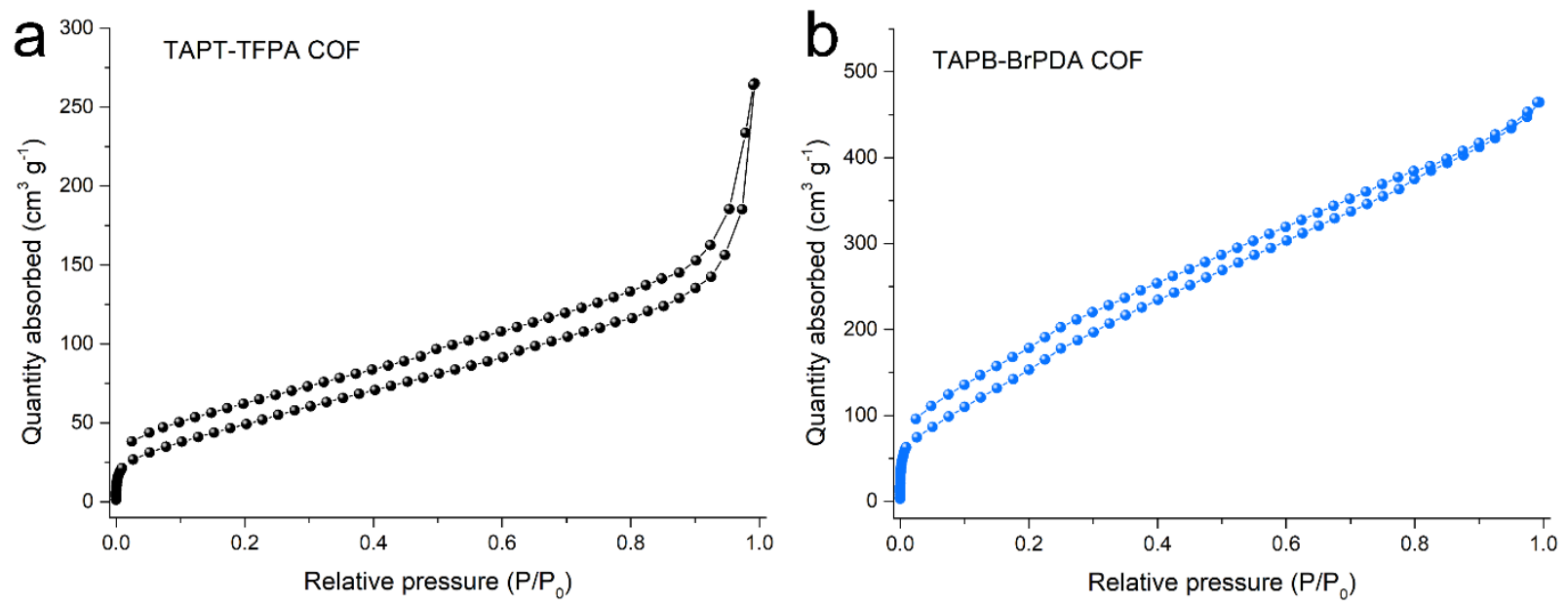

Figure S10. Nitrogen sorption isotherms for crystalline TAPA-TFPA COF aerogels and crystalline TAPB-BrPDA COF aerogels. 


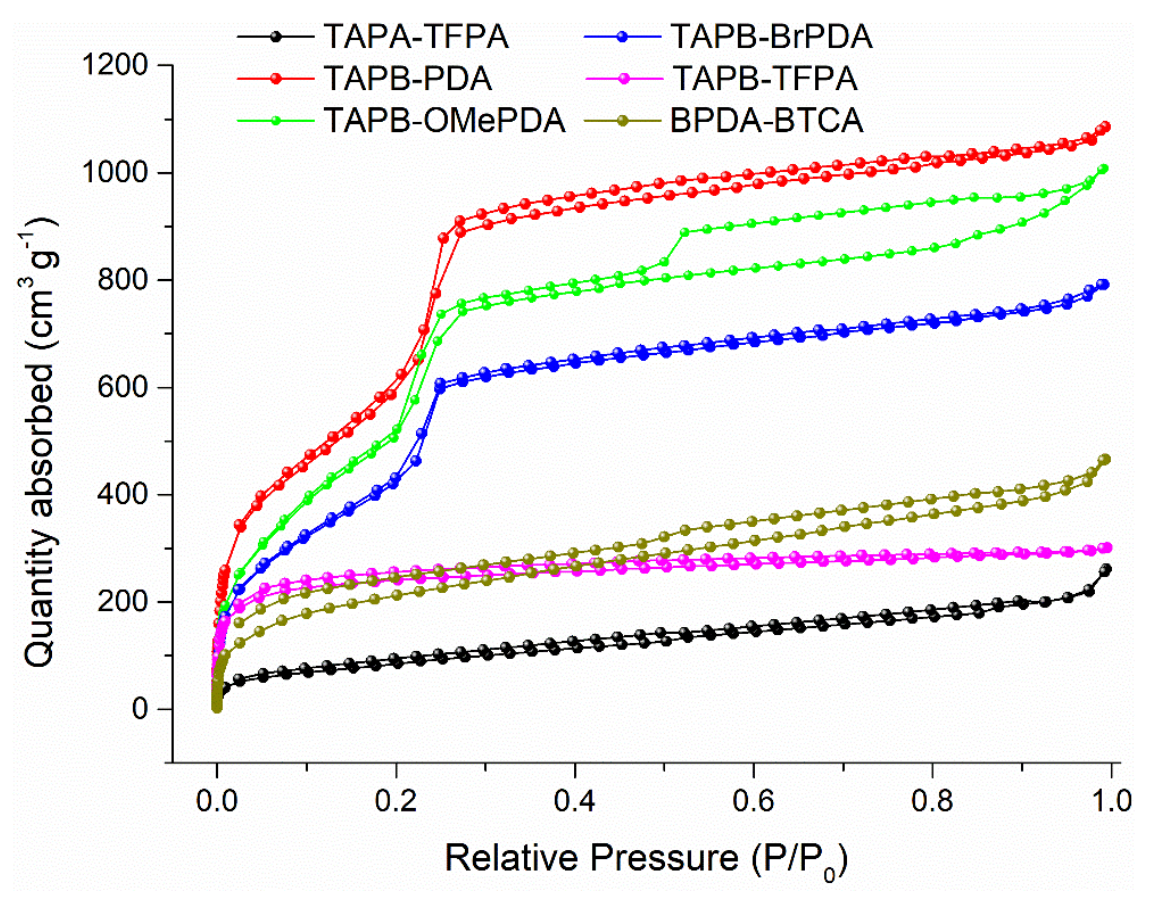

Figure S11. Nitrogen sorption isotherms for six different COF powders.

The BET surface areas for these COFs are $308 \mathrm{~m}^{2} / \mathrm{g}$ (TAPA-TFPA), $2264 \mathrm{~m}^{2} / \mathrm{g}$ (TAPB-PDA) $1992 \mathrm{~m}^{2} / \mathrm{g}$ (TAPB-OMePDA) $1619 \mathrm{~m}^{2} / \mathrm{g}$ (TAPB-BrPDA) $814 \mathrm{~m}^{2} / \mathrm{g}$ (TAPB-TFPA) and $776 \mathrm{~m}^{2} / \mathrm{g}$ (BPDA-BTCA), respectively. 

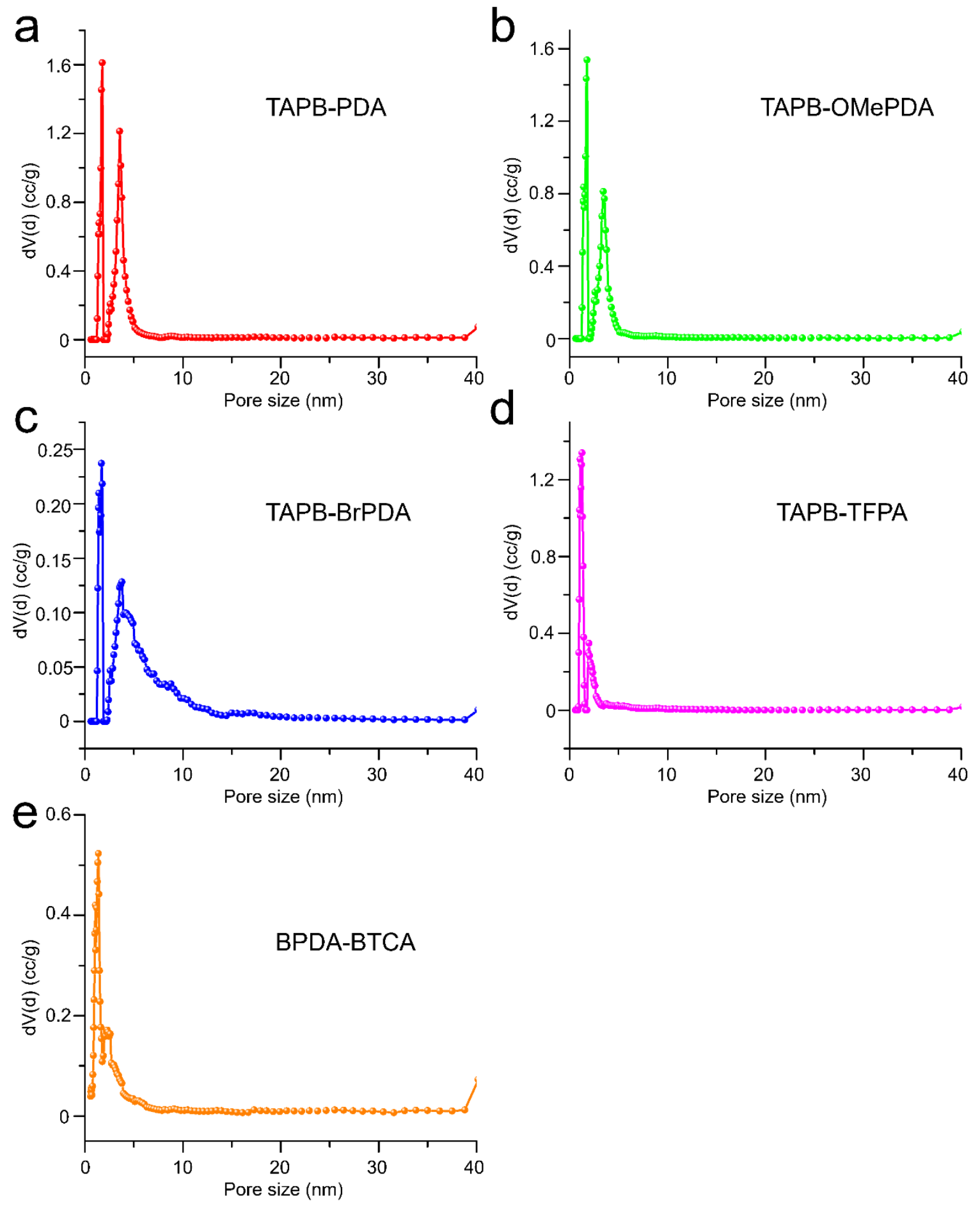

Figure S12. Pore size distributions for various COF aerogels calculated using NLDFT method. (a) TAPB-PDA, (b) TAPB-OMePDA, (c) TAPB-BrPDA, (d) TAPB-TFPA, (e) BPDA-BTCA 


\section{SEM analysis}

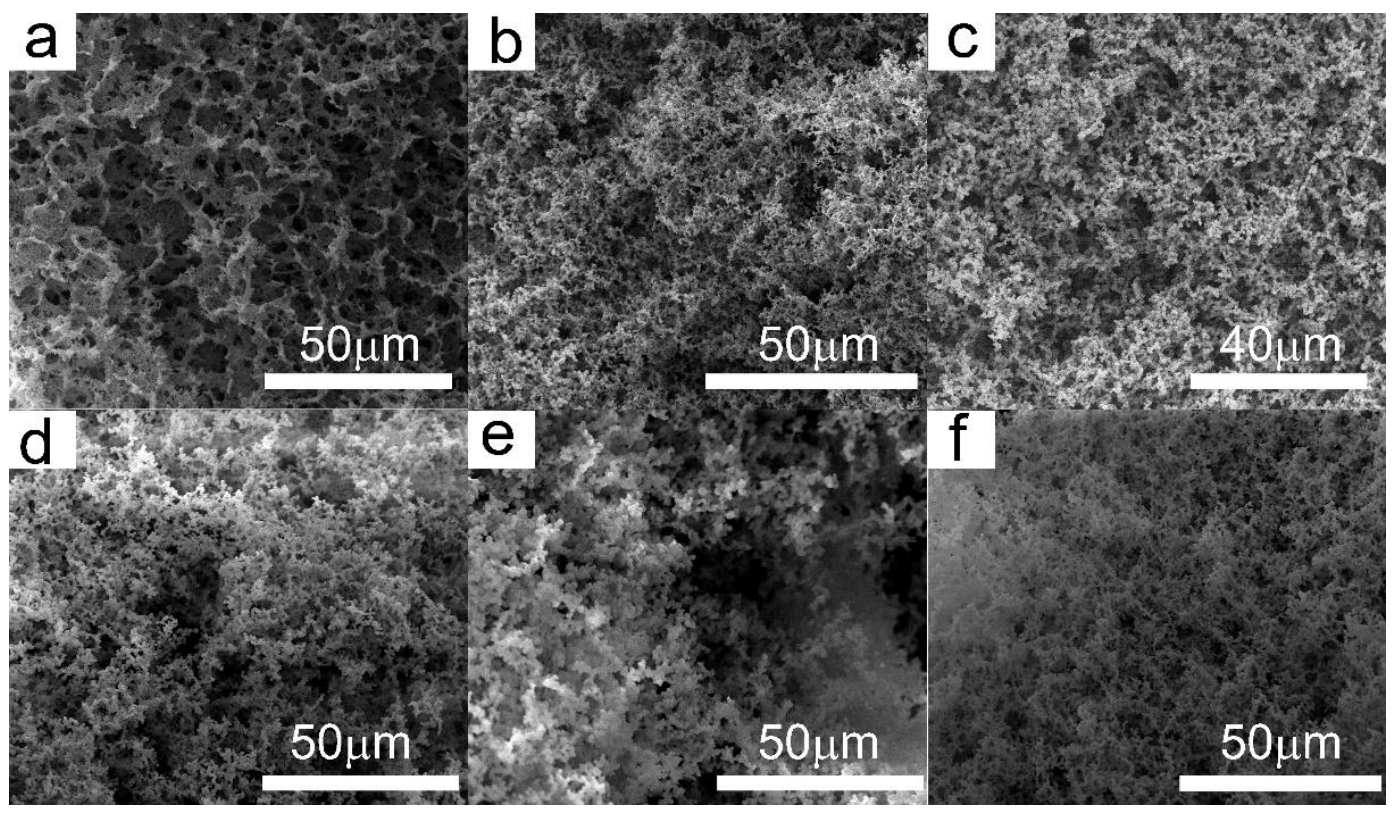

Figure S13. SEM micrographs of six crystalline COF aerogels. (a) TAPA-TFPA (b) TAPB-PDA (c)TAPB-OMePDA (d)TAPB-BrPDA (e) TAPB-TFPA (f)BPDA-BTCA

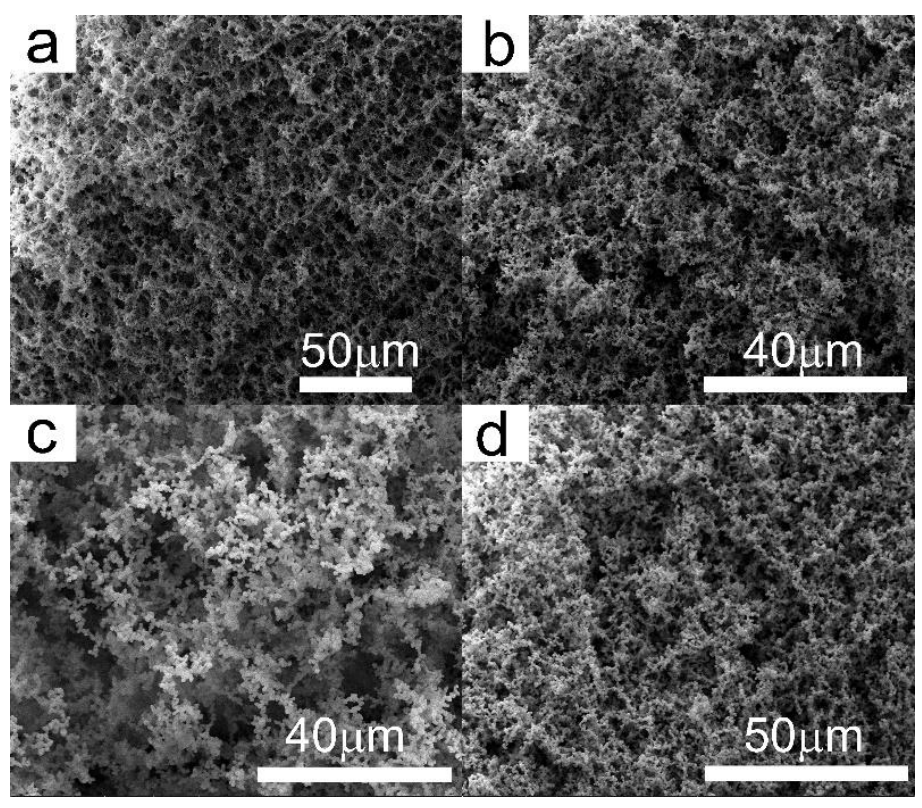

Figure S14. SEM micrographs of four amorphous aerogels before reactivation. (a) TAPA-TFPA (b) TAPB-PDA (c)TAPB-BrPDA (d)BPDA-BTCA 
6. Mechanical properties of COF aerogels

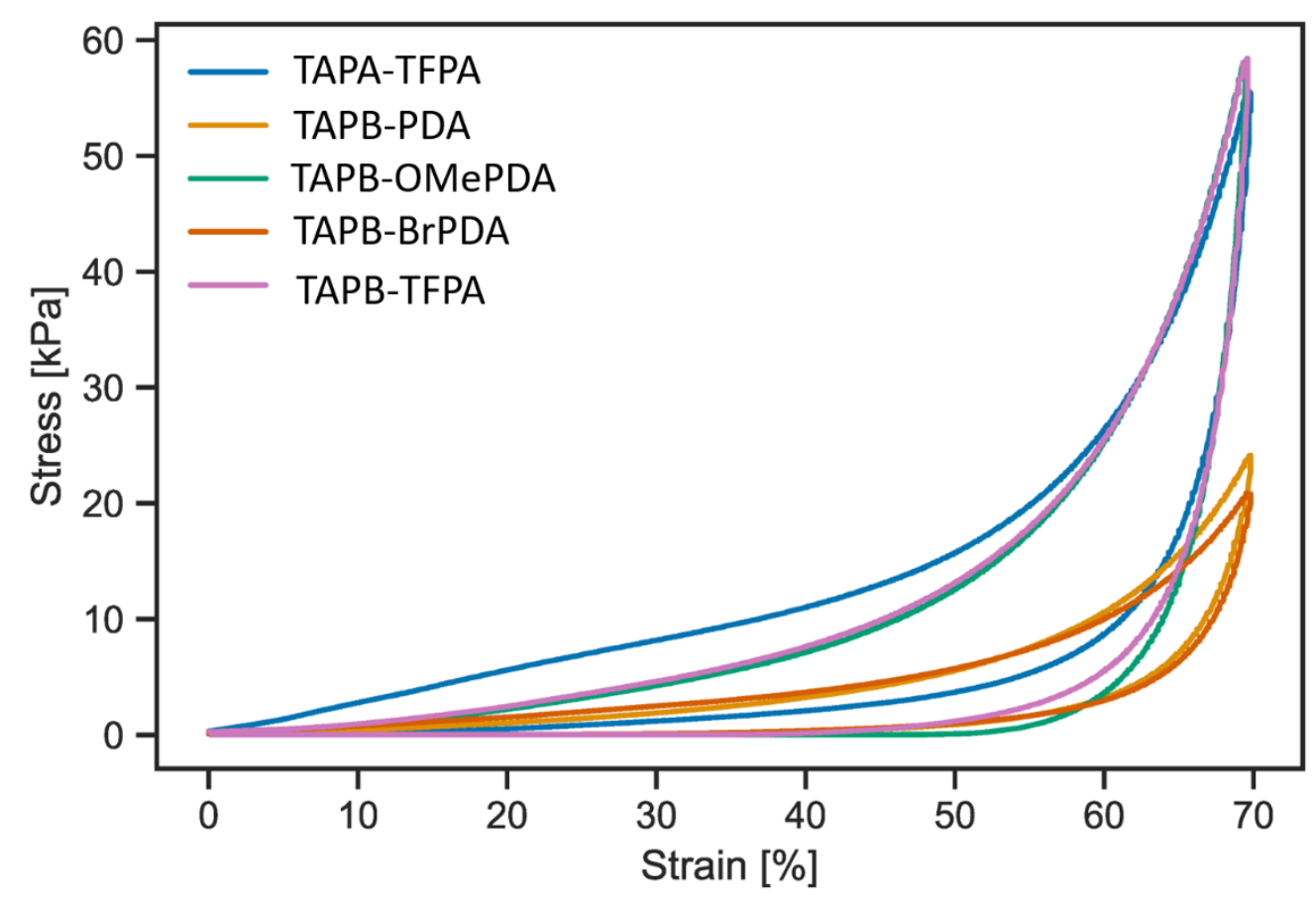

Figure S15. Stress-strain curves for selected COF aerogels.



Figure S16. Digital photographs of some COF aerogels before, during and after compression test 


\section{Studies of reaction conditions using TAPB-PDA COF as an example}

\section{(1) Solvent effect}

We studied the solvent effect on the gelation of COFs. DMSO could produce homogeneous gels. We were able to prepare gels with different sizes in $2 \mathrm{~mL}$ small-size vial, $4 \mathrm{~mL}$ medium-size vial and $25 \mathrm{~mL}$ large-size vial. All these hazy gels were homogeneous and uniform.

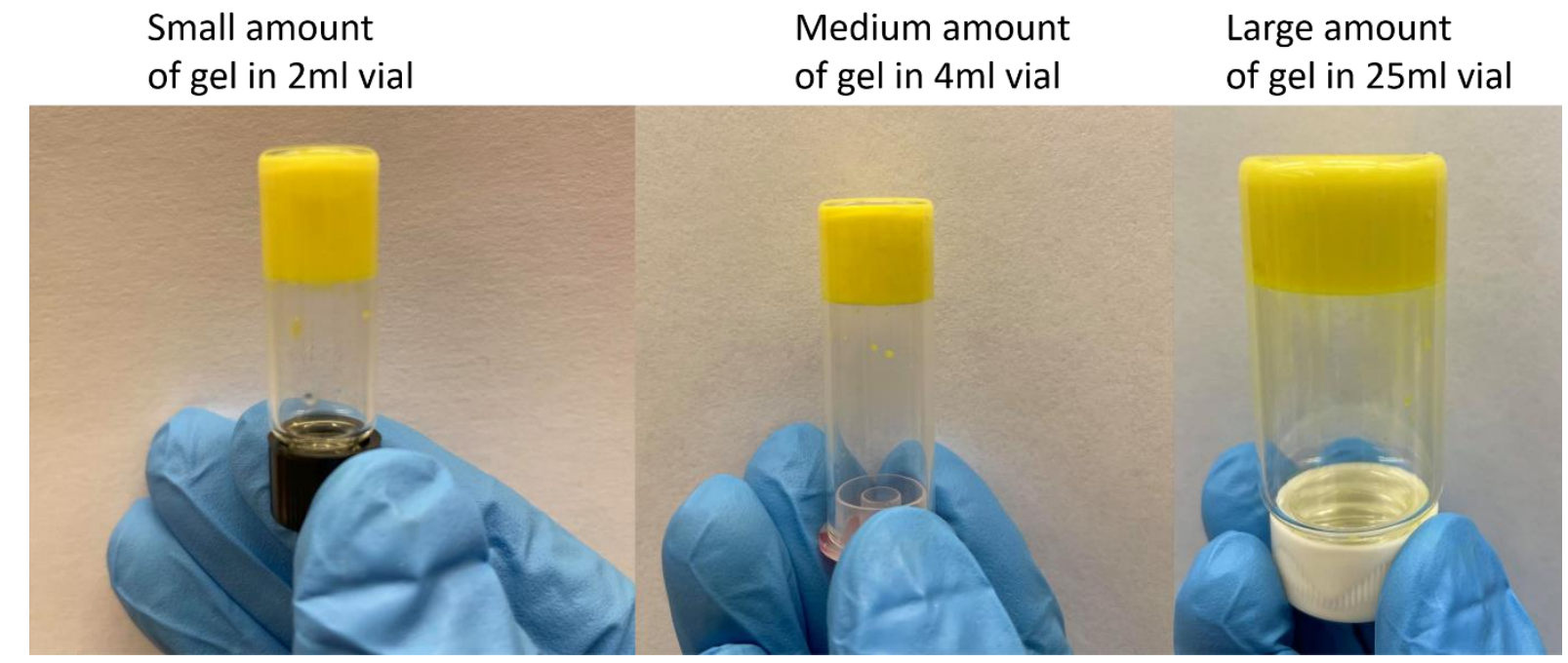

Figure S17. Wet COF gels prepared in different size glass vials at room temperature.

Other common solvents including dioxane, mesitylene, chloroform, THF, acetone and ethanol, and protic solvents including dimethylacetamide (DMAc), dimethylformamide (DMF), and N-methyl-2-pyrrolidone (NMP) were further studied to see whether these solvents help form COF gels.

As shown in Figure S16, solvents including dioxane, mesitylene, chloroform, THF, acetone and ethanol did not help COF monomer solution form gels. Only heterogeneous particles existed in the system. 

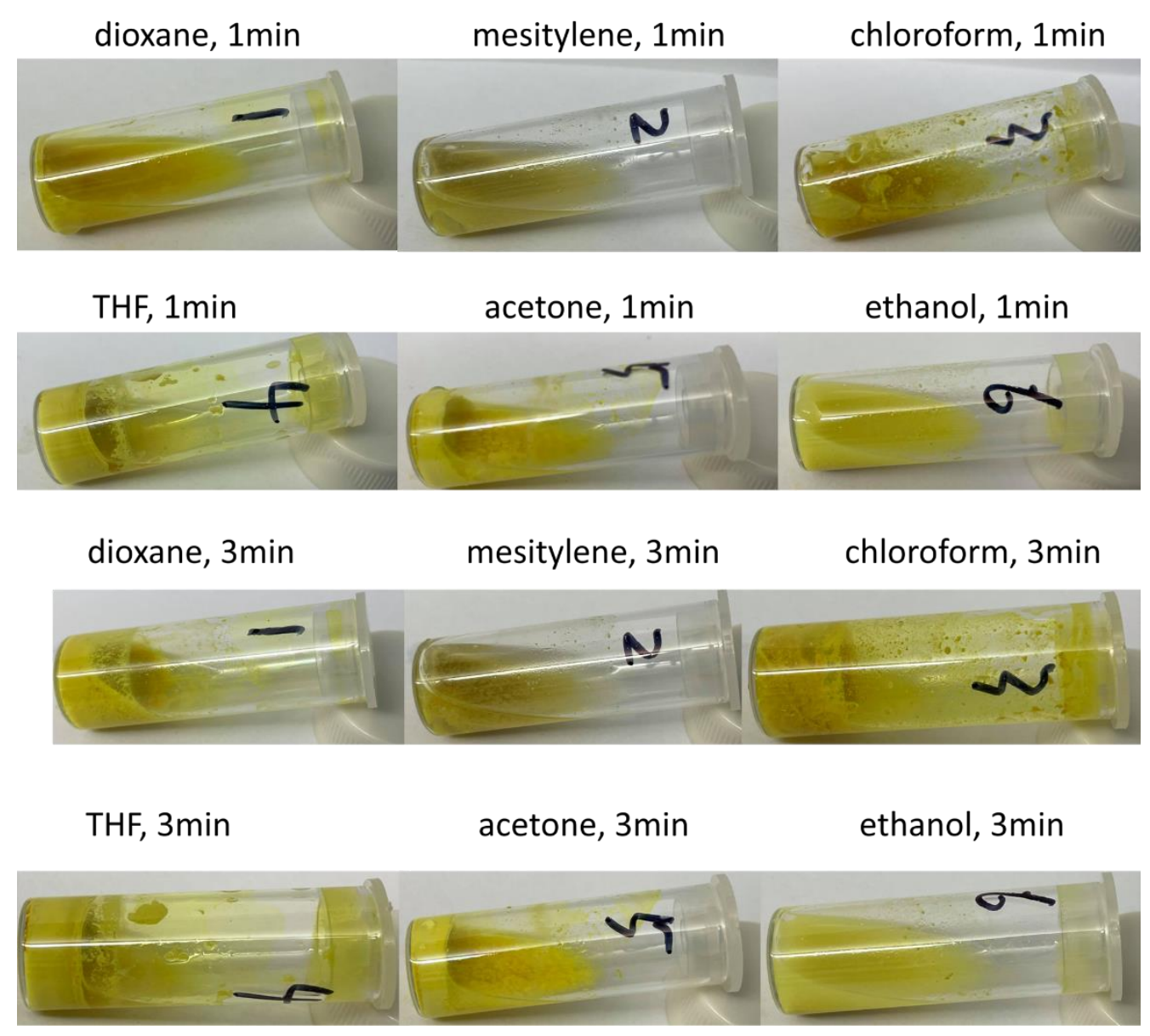

Figure S18. COF monomers reacted in different solvents. Reaction conditions: TAPB=21.09 mg, $\mathrm{PDA}=12.07 \mathrm{mg}, 1 \mathrm{~mL}$ various solvents in each vial, $0.1 \mathrm{~mL} 6 \mathrm{M} \mathrm{AcOH}$, room temperature.

We found that protic solvents helped TAPB-PDA COF form gels (see Figure S17). DMF, DMAc and NMP could all help TAPB-PDA COF form gels within one minute at room temperature. However, these solvents did not help TAPB-OMePDA COF form gels, indicating a low generality in the synthesis of COF aerogels. 


\section{TAPB-PDA COF}

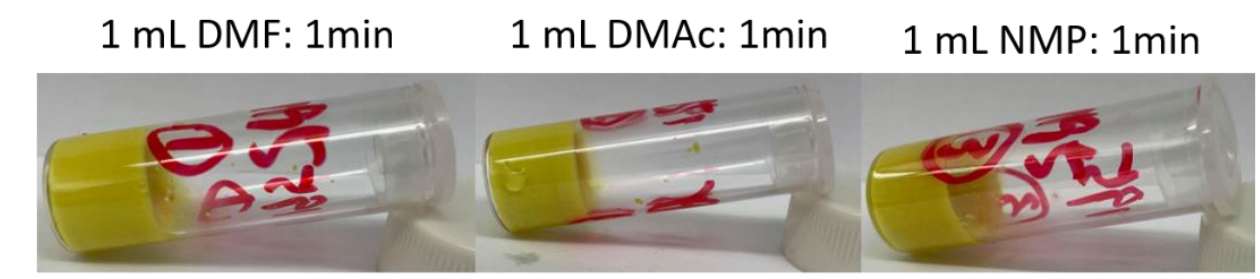

\section{TAPB-OMePDA COF}

$1 \mathrm{~mL} \mathrm{DMF:} 1 \mathrm{~min}$

$1 \mathrm{~mL}$ DMAc: $1 \mathrm{~min}$

$1 \mathrm{~mL} \mathrm{NMP:} 1 \mathrm{~min}$

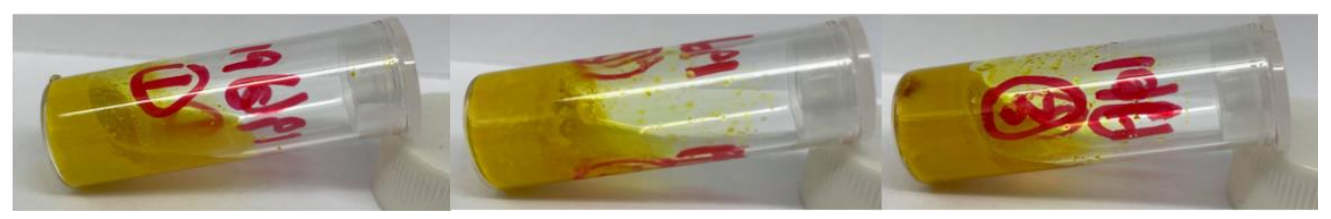

Figure S19. Exploration of solvent effects on COF gelation by using protic solvents including DMF, DMAc and NMP. Reaction conditions: TAPB $=21.09 \mathrm{mg}, \mathrm{PDA}=12.07 \mathrm{mg}$ or $\mathrm{OMePDA}=17.48 \mathrm{mg}, 1 \mathrm{~mL}$ various solvents in each vial, $0.1 \mathrm{~mL} 6 \mathrm{M} \mathrm{AcOH}$, room temperature.

\section{(2) Temperature}

We further studied the temperature effect on the final COF aerogel. The COF gels were reacted in DMSO solvents with all other reaction conditions $(\mathrm{TAPB}=21.09 \mathrm{mg}, \mathrm{PDA}=12.07 \mathrm{mg}, 1 \mathrm{~mL}$ DMSO, $0.1 \mathrm{~mL} 6 \mathrm{M}$ $\mathrm{AcOH})$ identical except the temperature. Each sample were incubated at room temperature, $80{ }^{\circ} \mathrm{C}$ and $160{ }^{\circ} \mathrm{C}$. We found that samples incubated at room temperature (amorphous aerogel weight is $20.1 \mathrm{mg}$ ) has a lower yield than those incubated at $80{ }^{\circ} \mathrm{C}$ (amorphous aerogel weight is $27.6 \mathrm{mg}$ ), indicating the COF monomers did not fully reacted compared to those reactions at higher temperature. However, samples reacted at higher temperature shrink due to the evaporation of DMSO at higher temperature.

\section{(3) Monomer Concentration}

Monomer concentrations also affected the gelation process. We took TAPB-PDA COF as an example and altered the monomer concentrations from low to high. As shown in Figure S18, lower concentration $(\mathrm{TAPB}=5.5 \mathrm{mg}, \mathrm{PDA}=3.02 \mathrm{mg}$ ) did not form gels as the solution was too dilute to form a connected system. 
Higher concentration $(\mathrm{TAPB}=42.18 \mathrm{mg}, \mathrm{PDA}=24.14 \mathrm{mg}$ ) of monomers formed gel, but the gel was not as homogeneous as those fabricated using the medium concentration (TAPB=21.09 $\mathrm{mg}, \mathrm{PDA}=12.07 \mathrm{mg}$ ). Furthermore, the gels prepared using higher concentration had similar volume as those prepared using lower concentration, indicating an increased density. So, both lower concentration and higher concentration are not optimized conditions for synthesis of COF gels.

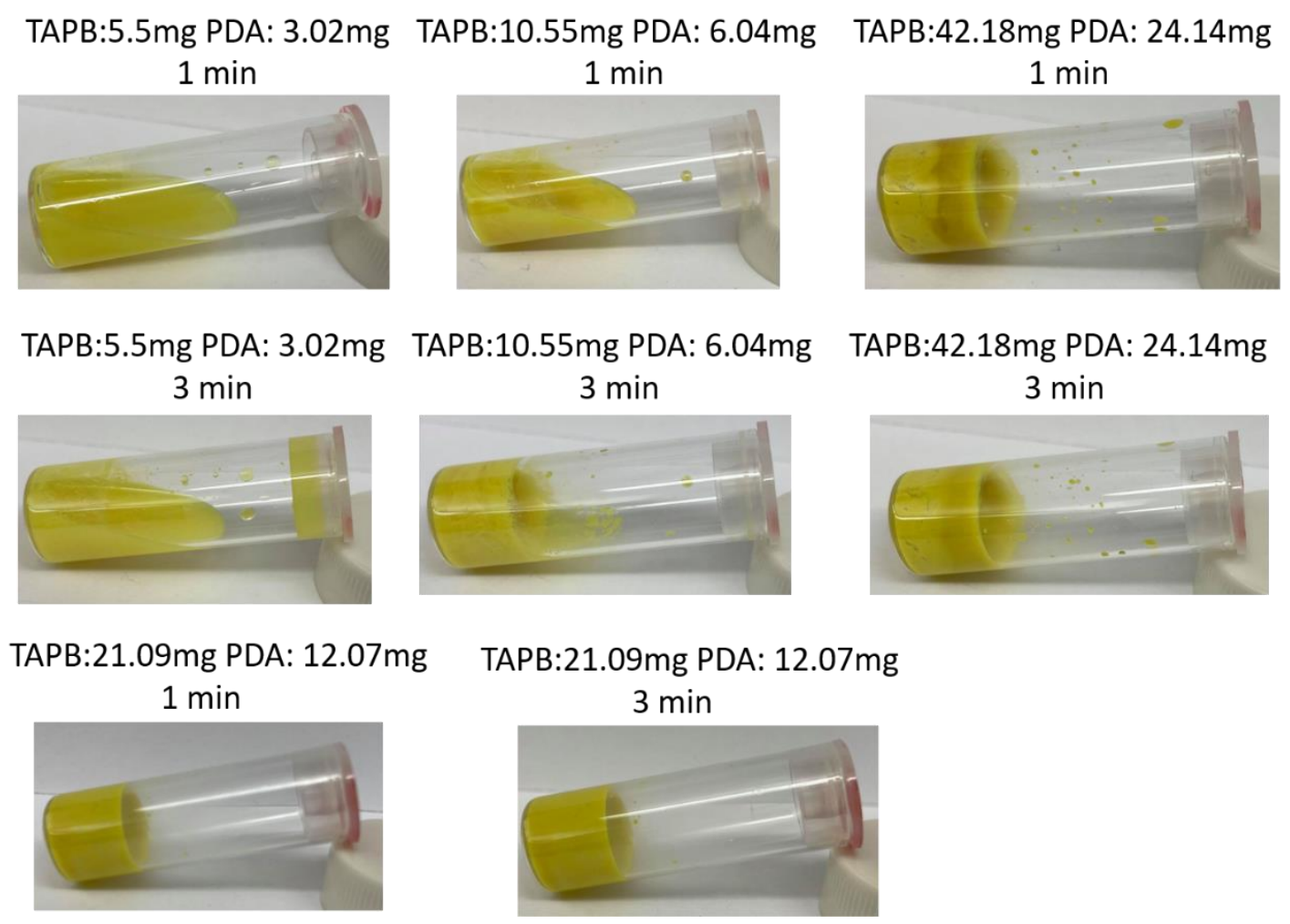

Figure S20. Exploration of the effects of monomer concentration on COF gelation in DMSO solvent by using TAPB-PDA COF as an example. Reaction conditions: 1mL DMSO, $0.1 \mathrm{~mL} 6 \mathrm{M}$ AcOH, room temperature.

\section{(4) Catalyst amounts}

We further studied the effects of catalyst amounts on the gelation by using TAPB-PDA COF as an example (see Figure S19). When no 6M AcOH was added, the solution did not form gels within one minute. It became cloudy slowly and form COF particles finally. When $0.05 \mathrm{~mL} 6 \mathrm{M} \mathrm{AcOH}$ was added, the solution 
gelled within one minute, but it did not form gels completely. After two minutes, the solution become gels completely. When $0.1 \mathrm{~mL} 6 \mathrm{M}$ AcOH was added, the monomer solution form homogenous gels within one minute. However, when more catalysts were added $(0.2 \mathrm{~mL}$ or $0.4 \mathrm{~mL}$ or $0.6 \mathrm{~mL} 6 \mathrm{M} \mathrm{AcOH})$, the solution formed heterogeneous gels. The texture of the COF gels became rougher with the increasing amounts of acetic acid.
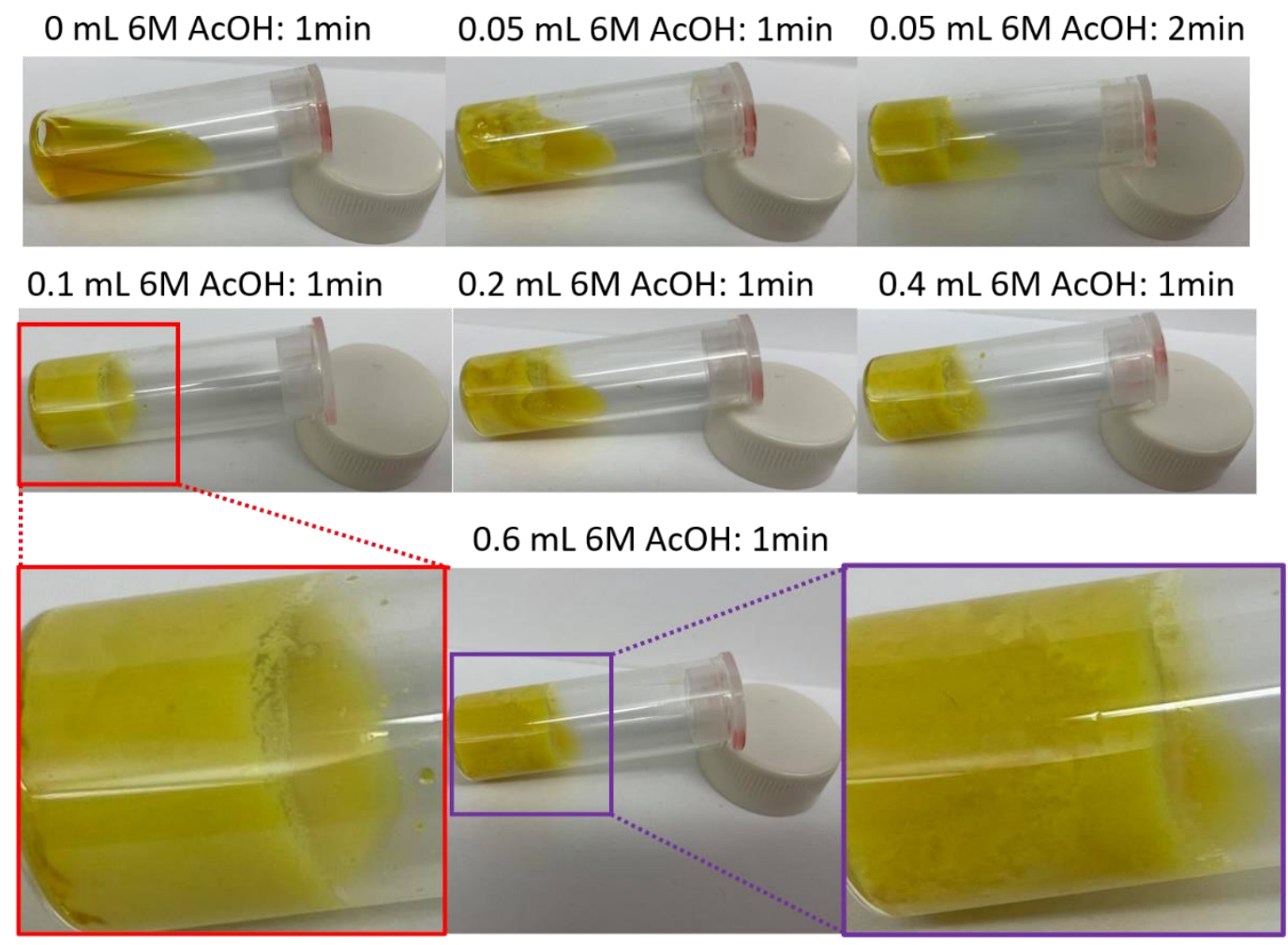

Figure S21. Exploration of the effects of catalyst amounts on COF gelation in DMSO solvent by using TAPB-PDA COF as an example. Reaction conditions: 1mL DMSO, TAPB=21.09 mg, PDA=12.07 mg, room temperature. 


\section{(6) Freeze drying}

We studied the effect of freeze drying for the six COFs since freeze drying is more easily accessible compared to supercritical $\mathrm{CO}_{2}$ drying. After reaction in DMSO, all wet gels were thoroughly washed using THF, acetone, ethanol and water. Solvent exchange with water is important for the followed freeze drying procedure. Gels filled with water were frozen using liquid nitrogen and were freeze dried for 12 hours. PXRD analyses showed that TAPA-TFPA, TAPBPDA, TAPB-BrPDA and BPDA-BTCA COFs were amorphous after reaction in DMSO, through washings and freeze drying (Figure S20). TAPB-OMePDA COF showed good crystallinity and TAPB-TFPA COF showed a low crystallinity (Figure S20).
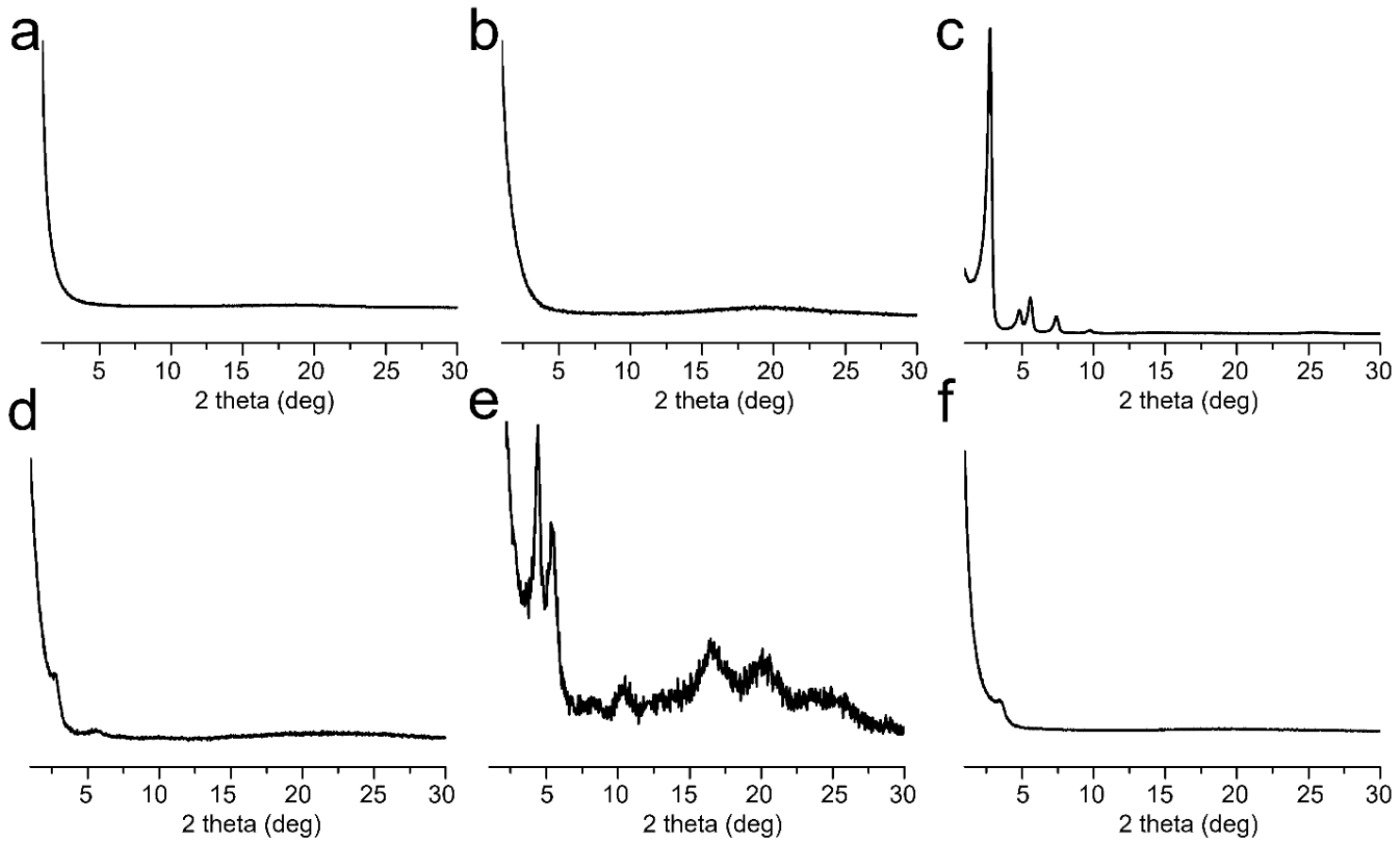

Figure S22. PXRD spectra for dry COF gels after reaction in DMSO, thorough washings and freeze drying. (a) TAPA-TFPA (b) TAPB-PDA (c) TAPB-OMePDA (d) TAPB-BrPDA (e) TAPB-TFPA (f) BPDA-BTCA 
After reactivation of amorphous COF gels in dioxane and mesitylene, a second step drying procedure has to be taken. Freeze drying is not an effective method in this step. Water used in freeze drying process has a high surface tension, so pore collapse occurs for fragile COFs during the drying process. ${ }^{2}$ So, those fragile COFs lose partial crystallinity during the freeze drying process (Figure S21). Two COFs (TAPB-PDA and BPDA-PDA) were dried using $\mathrm{ScCO}_{2}$ before reaction in dioxane and mesitylene, and were further dried using freeze drying in the second step after reaction in dioxane and mesitylene. These two COFs showed very limited crystallinity due to the pore collapse (Figure S21). However, if $\mathrm{ScCO}_{2}$ drying was employed in the second step, crystalline COFs could be produces as shown in Figure 2.

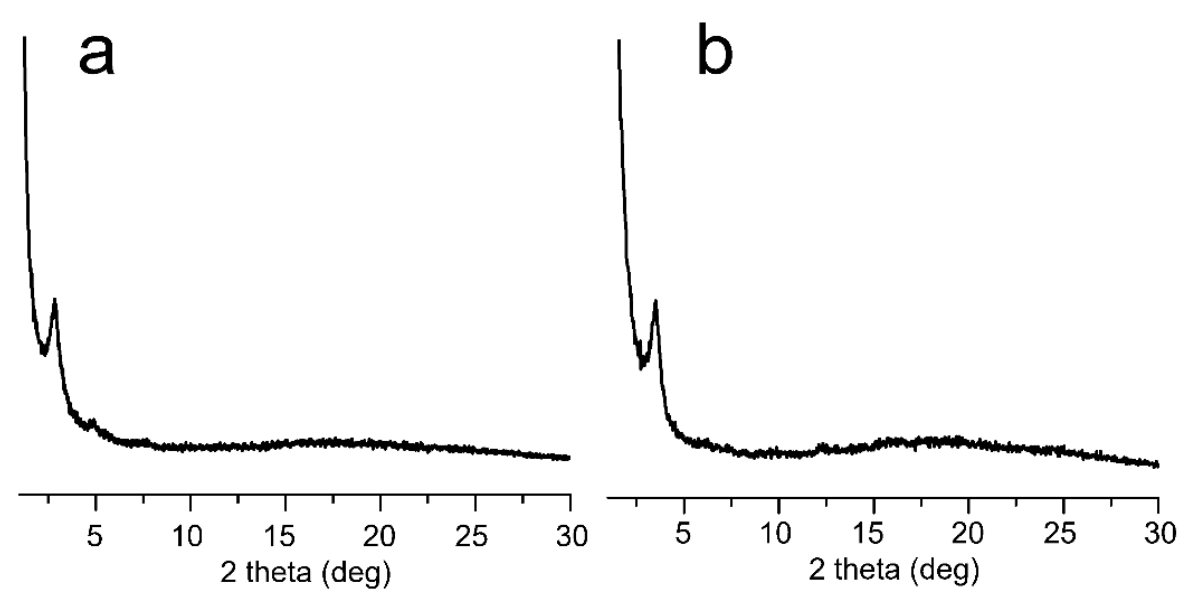

Figure S23. PXRD spectra for TAPB-PDA and BPDA-PDA COF aerogels after freeze drying.

For those amorphous COF gels after freeze drying in the first step (see Figure S20), they gained crystallinity after reaction in dioxane/mesitylene and supercritical $\mathrm{CO}_{2}$ drying in the second step (Figure S22). 


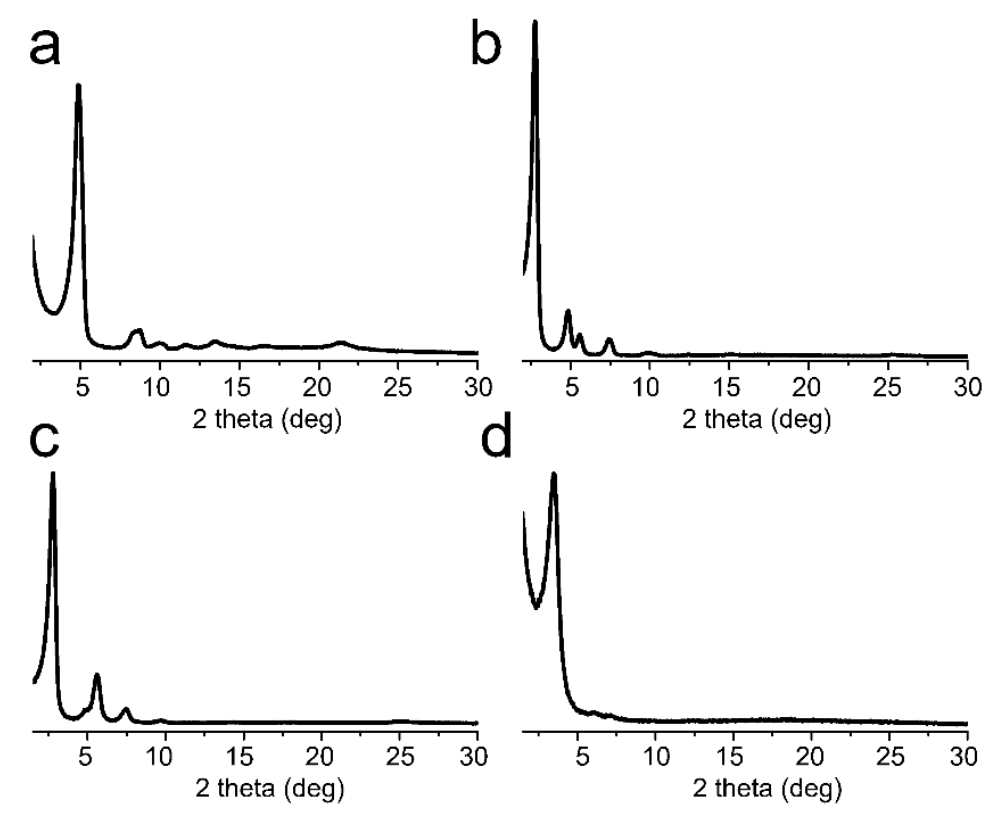

Figure S24. PXRD spectra for the reactivated gels after second-step supercritical $\mathrm{CO}_{2}$ drying. In detail, those amorphous gels produced after freeze drying in the first step were further reacted in dioxane and mesitylene to recover crystallinity. After reaction, they were dried using supercritical $\mathrm{CO}_{2}$ drying in the second step. (a) TAPA-TFPA (b) TAPB-PDA (c) TAPB-BrPDA (d) BPDA-BTCA 


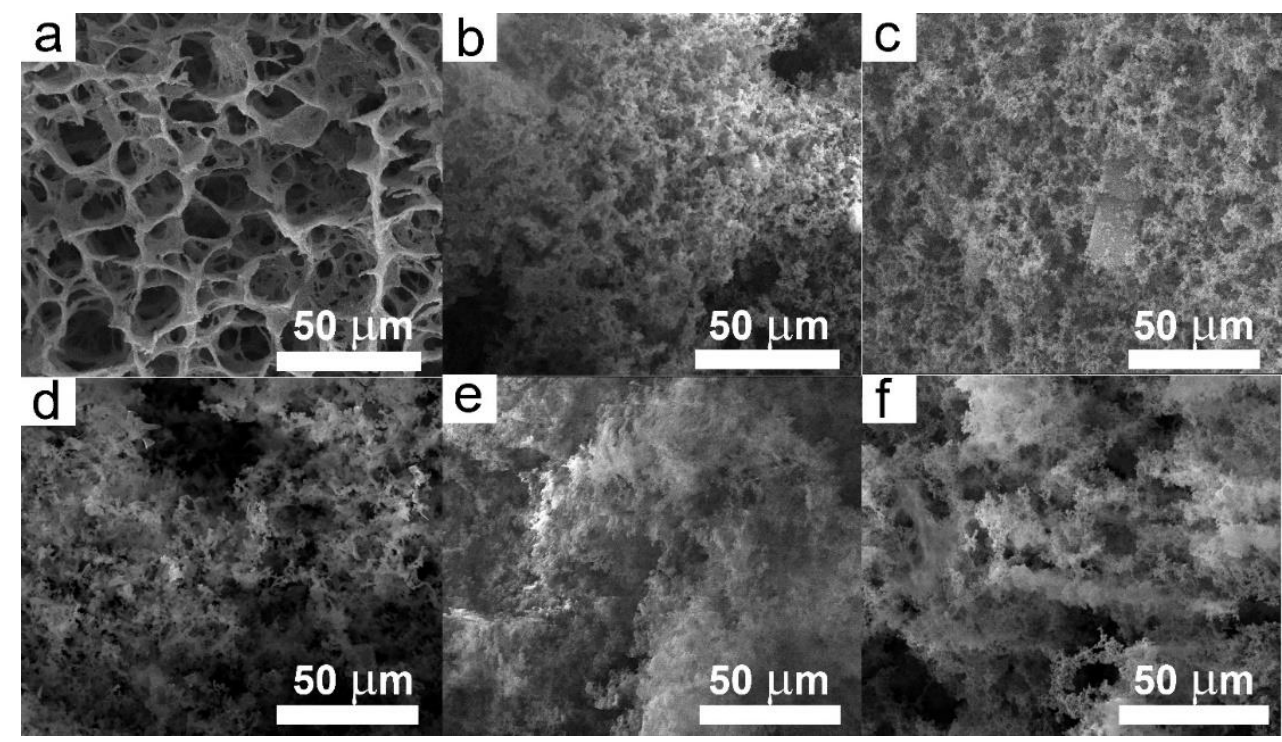

Figure S25. SEM graphs for crystalline COF gels synthesized with freeze drying involved in the first step and supercritical $\mathrm{CO}_{2}$ drying used in the second step. (a) TAPA-TFPA (b) TAPB-PDA (c) TAPB-OMePDA (d) TAPB-BrPDA (e) TAPB-TFPA (f) BPDA-BTCA. TAPB-OMePDA and TAPBTFPA COFs gained crystallinity after first step and no second step was employed for them.

We also analyzed the morphologies of crystalline COF aerogels synthesized by using freeze drying in the first step and supercritical $\mathrm{CO}_{2}$ drying in the second step. For TABP-OMePDA and TAPB-TFPA COFs, no second step was used since they could gain crystallinity directly in the first step. The morphologies of these COF aerogels were very similar to those samples prepared using supercritical $\mathrm{CO}_{2}$ drying in both first and second steps, indicating that drying method did not affect the morphologies of COF aerogels significantly. For TAPA-TFPA COF, it showed a more regular honeycomb-type pores (Figure S23a, S24a), which is caused by the ice. TAPA-TFPA COF aerogel after supercritical $\mathrm{CO}_{2}$ drying also displayed a honeycomb-type pores (Figure 2i, S13-14), but those pores were not as apparent and regular as those after freeze drying. 


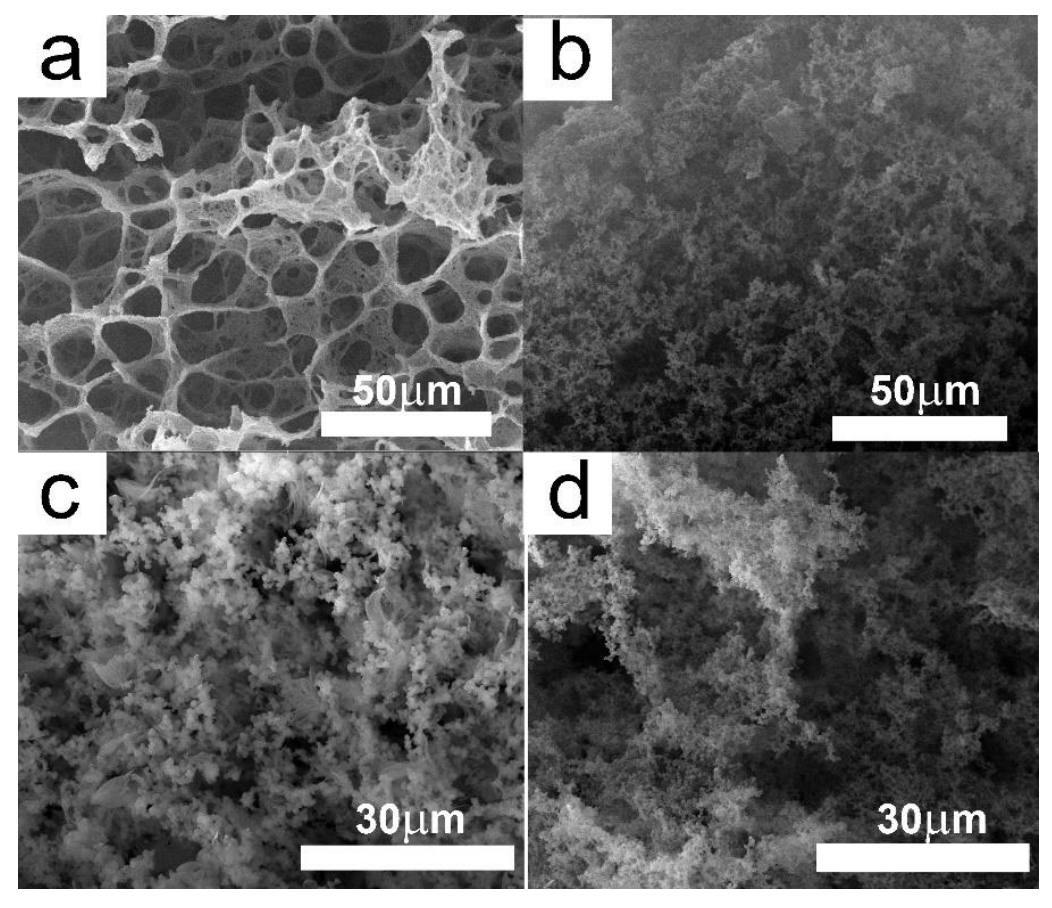

Figure S26. SEM graphs for amorphous gels synthesized after freeze drying in the first step. (a) TAPA-TFPA (b) TAPB-PDA (c)TAPB-BrPDA (d) BPDA-BTCA.

\section{Organic solvents absorption}

Absorption measurement. $\mathrm{COF}$ aerogels after $\mathrm{ScCO}_{2}$ drying were used in the absorption measurement directly without further treatment. Aerogel samples were immersed in the different organic solvent separately for $5 \mathrm{~min}$ at the room temperature. The weight was recorded before (W1) and after (W2) absorption to calculate the weight gain. The absorption capacity of COF aerogels was calculated using the equation $(\mathrm{W} 2-\mathrm{W} 1) / \mathrm{W} 1 \times 100 \%$. The recyclability test was performed for chloroform by repeating the absorption process after solvent exchange using ethanol and supercritical $\mathrm{CO}_{2}$ drying. 


\section{Organic dye removal}

General procedure for absorption study: The absorption study was performed with a modified procedure according to the previous report. ${ }^{4}$ Briefly, to a $20 \mathrm{ml}$ vial, $2.5 \mathrm{mg}$ COF-aerogel was added into $4 \mathrm{~mL}$ stock pollutants solution. The concentration of pollutants solution is $10 \mu \mathrm{M}$ except $\mathrm{Au}$ NP solution was prepared of a higher concentration of $26.7 \mu \mathrm{M}$. For the removal efficiency study, reactions were stirred for 1 hour at room temperature (stirring rate of 400 r.p.m), and then the solution was filtered with a syringe having 0.22 um nylon membrane filter. The absorption of the filtered solution is measured by UV-vis spectroscopy (Agilent Cary-60). The pollutant removal efficiency was calculated based on the previous report. ${ }^{4}$ For the kinetic study, $2.5 \mathrm{mg}$ COF aerogel was first dissolved in $4 \mathrm{ml}$ water and then sonicated for 5 minutes until forming a uniform mixture. Then another $4 \mathrm{ml}$ pollutant stock was added to aerogel in order to get the final pollutant concentration of $10 \mu \mathrm{M}(26.7 \mu \mathrm{M}$ for Au NPs). The mixture was stirred at 400 r.p.m. $0.6 \mathrm{ml}$ sample was taken from the mixture at different time intervals and filtered with a syringe having 0.22 um nylon membrane filter. The absorption is measured by UV-vis spectroscopy. The pseudosecond-order rate constants $\left(K_{o b s}\right)$ were calculated using Langmuir model according to the previous report (ACS Omega 2019, 4, 22504-22513).

General procedure for flow-through adsorption studies: $5 \mathrm{mg}$ COF-aerogel was taken in 7.5 $\mathrm{mL}$ water and then it was kept for sonication for 15 minutes until forming a uniform. Then the mixture was packed inside a column and the COF-aerogel was treated as column bed for pollutants. Later, $5 \mathrm{~mL} 10 \mu \mathrm{M}$ MB solution was passed through the column bed in order to get the complete removal of pollutants. 


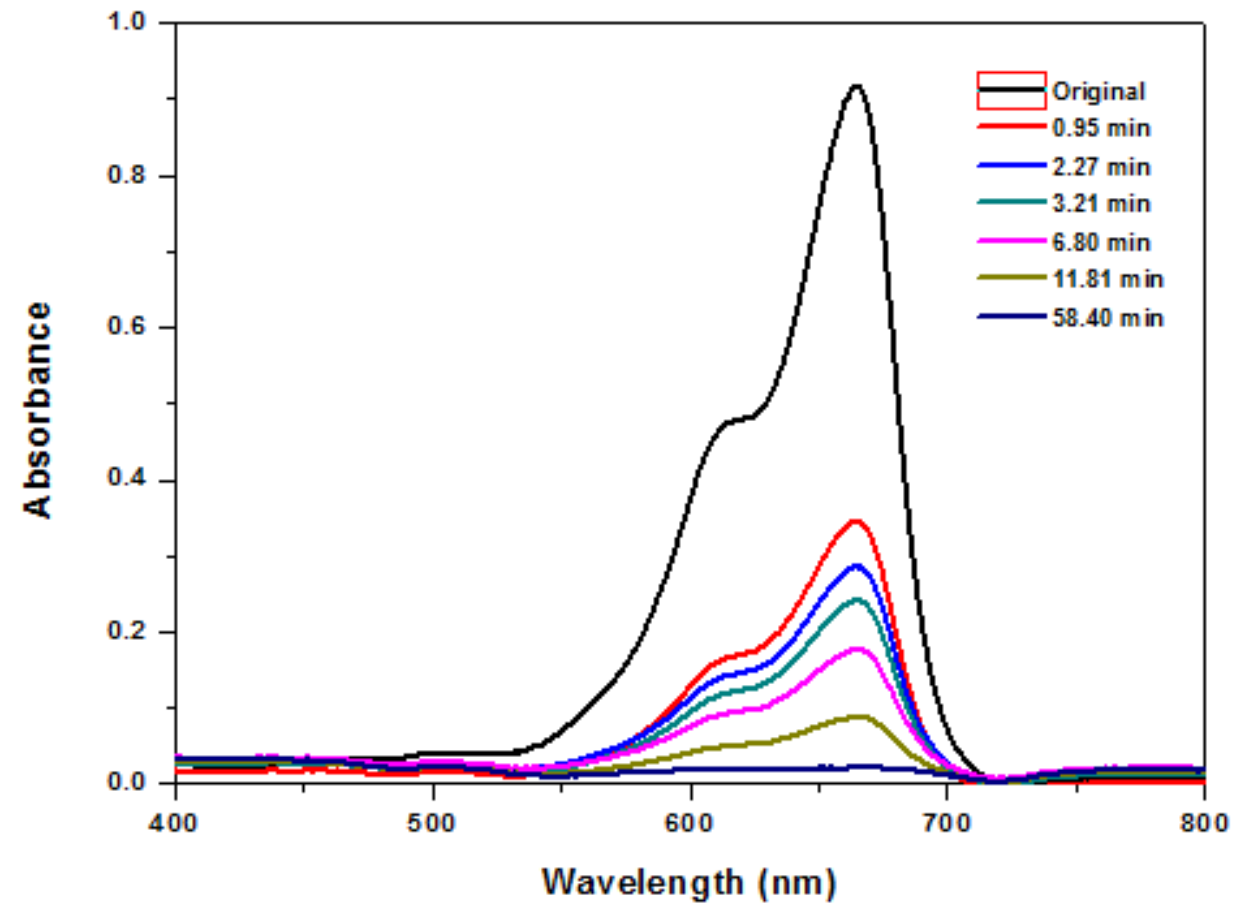

Figure S27.Time-dependent removal efficiency of Methylene Blue using TAPA-TFPA powder 

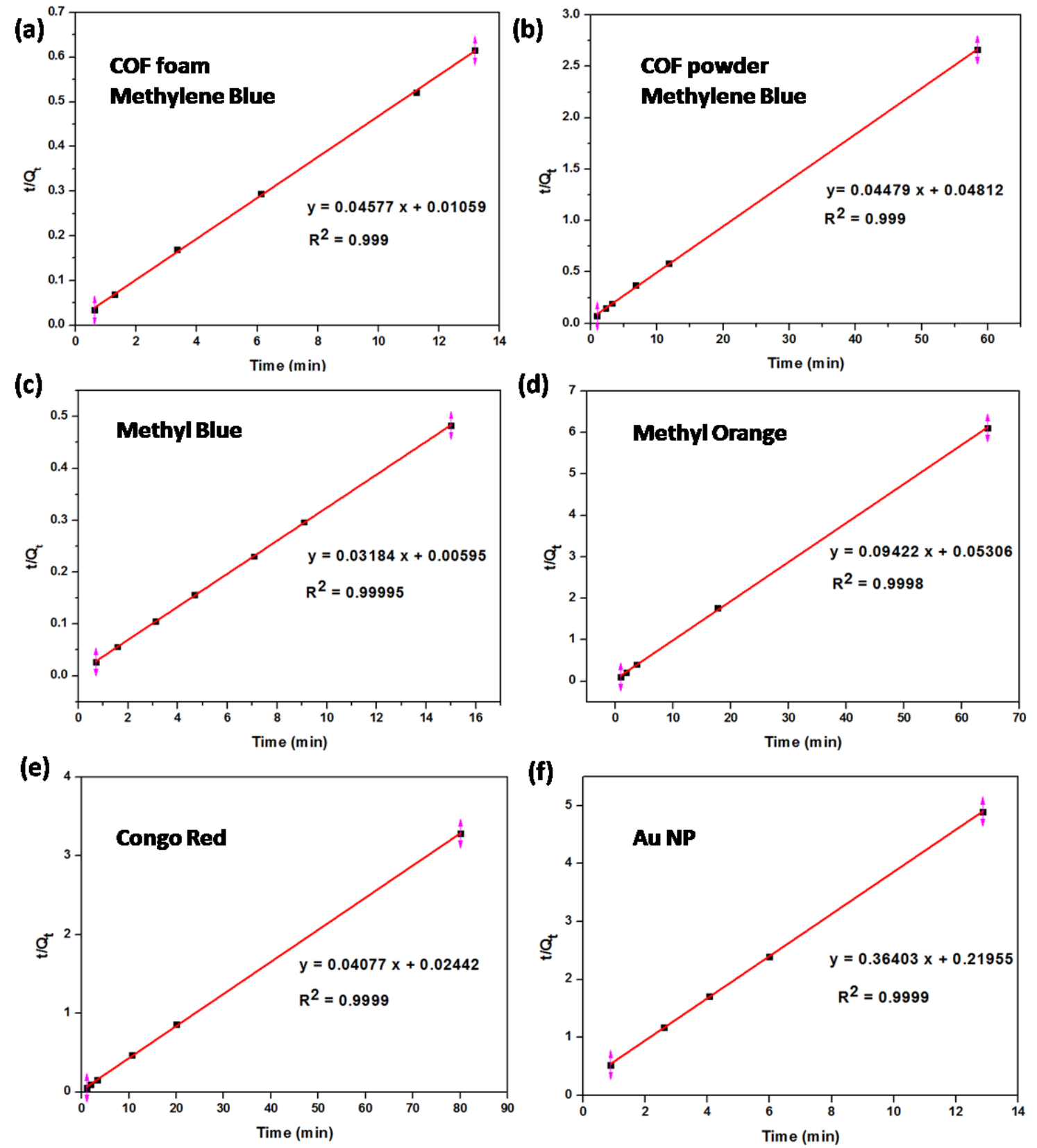

Figure S28. The rate of adsorption measured from the linear fit Langmuir plot for the adsorption of (a) Methylene Blue using TAPA-TFPA aerogel (b) Methylene Blue using TAPA-TFPA powder (c) Methyl Blue using TAPA-TFPA aerogel (d) Methyl Orange using TAPA-TFPA aerogel (e). Congo red using TAPA-TFPA aerogel (f) Au NPs using TAPA-TFPA aerogel 


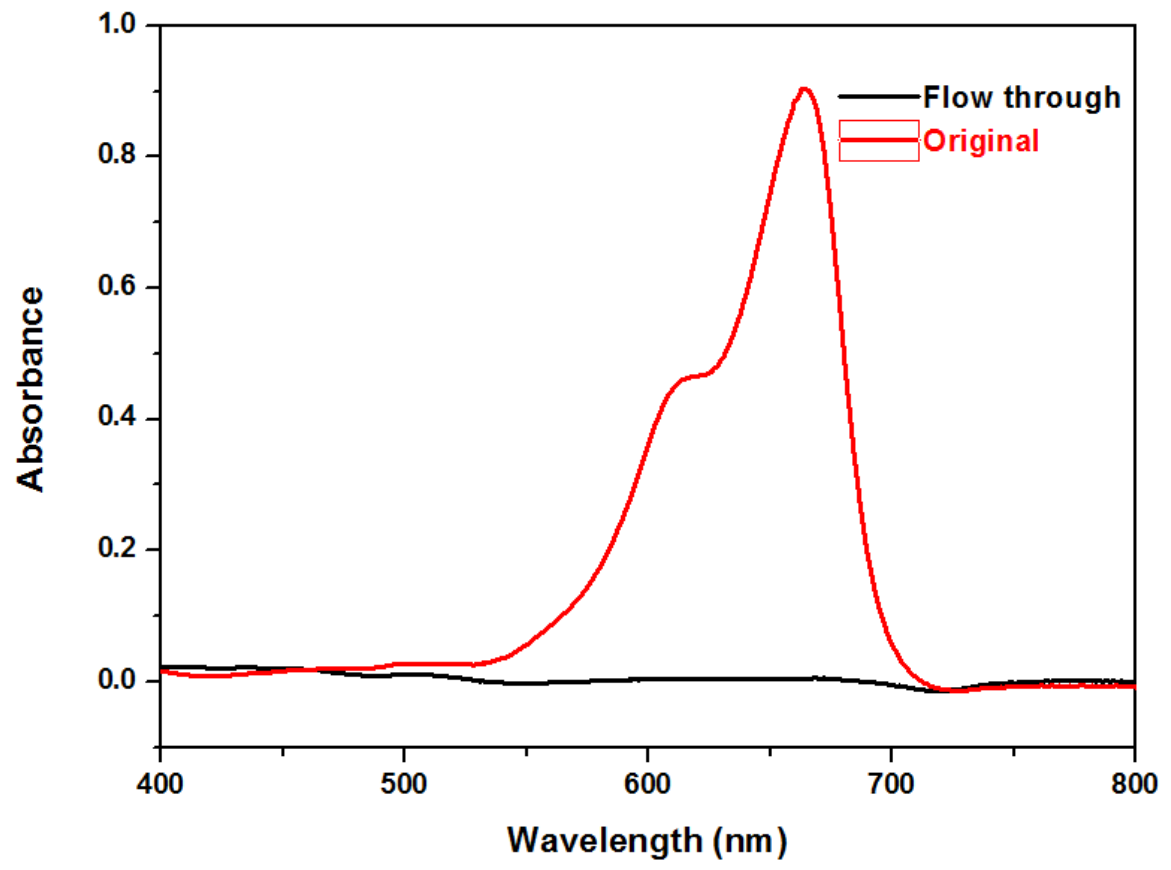

Figure S29. Flow-through experiment. Absorption of MB before (red) and after (black) passing through the COF column. 

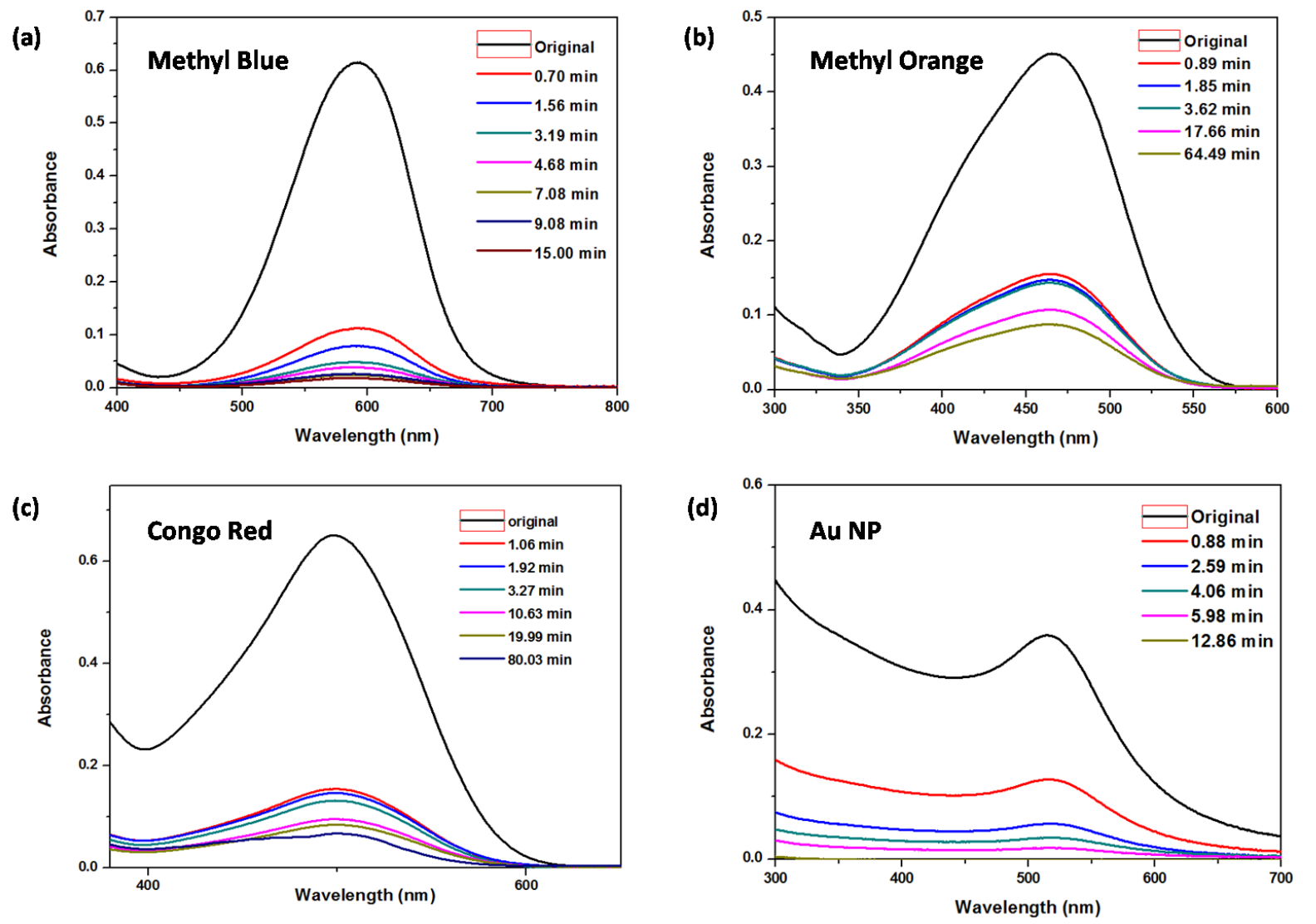

Figure S30. Time-dependent removal efficiency of (a) Methyl Blue (b) Methyl Orange (c) Congo Red (d) Gold nanoparticles using TAPA-TFPA aerogel. 

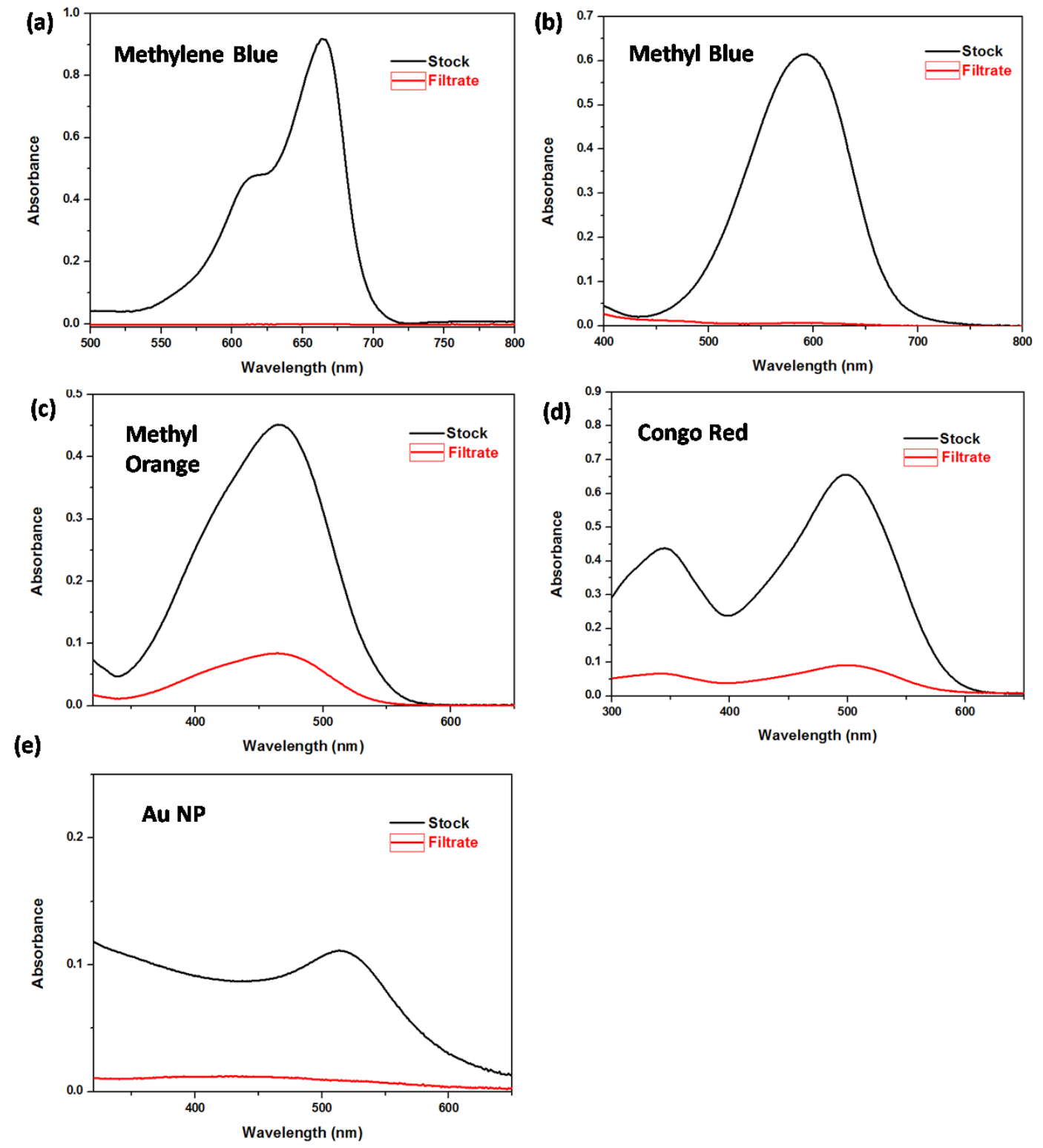

Figure S31. Pollutant removal efficiency of (a). Methylene Blue (b) Methyl Blue (c) Methyl Orange (d). Congo red (e) Au NPs using TAPA-TFPA aerogel 
Table S3. Rate of adsorption of various pollutants using TAPA-TFPA aerogel.

\begin{tabular}{|l|c|}
\hline Pollutants & $\mathbf{K}_{\text {obs }} \mathbf{( g . \mathbf { m g } ^ { - 1 } \mathbf { ~ m i n } ^ { - \mathbf { 1 } } )}$ \\
\hline Congo Red & 0.068 \\
\hline Methyl blue & 0.17 \\
\hline Methylene Blue & 0.20 \\
\hline Methylene Blue & 0.048 \\
\hline Using COF Powders & \\
\hline Methyl Orange & 0.167 \\
\hline Au NPs & 0.60 \\
\hline
\end{tabular}

\section{Iodine uptake}

Procedure for iodine vapor sorption. Similar weight of COF aerogel and powder samples were loaded in small open vials. The small open vials were placed in a $25 \mathrm{ml}$ large vial containing excess iodine (4 g). The large vial was sealed and kept in an oven at $75{ }^{\circ} \mathrm{C}$. To prevent the evaporation of iodine vapor, all large vials were further sealed using Teflon tape. After certain time intervals, the large vial was taken out and cooled down to room temperature. The small vial containing the COF sample was weighted and placed back into the iodine-containing large vial. The large vial was sealed and put back in the oven at $75{ }^{\circ} \mathrm{C}$ to continue the adsorption process until the mass of the small vial containing the COF sample did not change.

Procedure for iodine escape measurements. An open small $4 \mathrm{ml}$ vial containing the iodine loaded COF sample was placed in an empty large vial $(25 \mathrm{~mL})$. The large vial was sealed and placed under ambient conditions. After a certain period, the weight of the small vial containing the COF sample was recorded and the small vial was placed back into the empty large vial. The large vial was sealed to continue the desorption process. The iodine escape measurements were carried for a period of 7 days. 


\section{References}

(1) Zhai, L.; Huang, N.; Xu, H.; Chen, Q.; Jiang, D. A Backbone Design Principle for Covalent Organic Frameworks: The Impact of Weakly Interacting Units on CO2 Adsorption. Chem. Commun. 2017, 53 (30), 4242-4245.

(2) Zhu, D.; Verduzco, R. Ultralow Surface Tension Solvents Enable Facile COF Activation with Reduced Pore Collapse. ACS Appl. Mater. Interfaces 2020. 12, 29, 33121-33127.

(3) Bai, L.; Phua, S. Z. F.; Lim, W. Q.; Jana, A.; Luo, Z.; Tham, H. P.; Zhao, L.; Gao, Q.; Zhao, Y. Nanoscale Covalent Organic Frameworks as Smart Carriers for Drug Delivery. Chem. Commun. 2016, 52 (22), 4128-4131.

(4) Karak, S.; Dey, K.; Torris, A.; Halder, A.; Bera, S.; Kanheerampockil, F.; Banerjee, R. Inducing Disorder in Order: Hierarchically Porous Covalent Organic Framework Nanostructures for Rapid Removal of Persistent Organic Pollutants. J. Am. Chem. Soc. 2019, 141 (18), 7572-7581. 